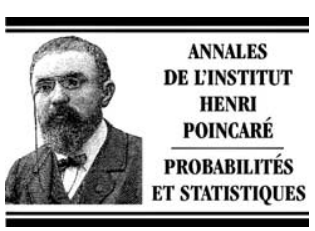

www.elsevier.com/locate/anihpb

\title{
A trivariate law for certain processes related to perturbed Brownian motions
}

\author{
Philippe Carmona ${ }^{\mathrm{a}}$, Frédérique Petit ${ }^{\mathrm{b}, *}$, Marc Yor $^{\mathrm{b}}$ \\ a Département de mathématiques, laboratoire Jean Leray UMR 6629, université de Nantes, 2, rue de la Houssinière, BP 92208 , \\ 44322 Nantes cedex 03, France \\ ${ }^{\mathrm{b}}$ Laboratoire de probabilités et modèles aléatoires, université Paris VI, casier 188, 4, place Jussieu, 75252 Paris cedex 05, France
}

Received 19 December 2002; accepted 21 November 2003

Available online 18 May 2004

\begin{abstract}
D. Williams' path decomposition and Pitman's representation theorem for BES(3) are expressions of some deep relations between reflecting Brownian motion and the 3-dimensional Bessel process.

In [Ph. Carmona et al., Stochastic Process. Appl. 7 (1999) 323-333], we presented an attempt to relate better reflecting Brownian motion and the 2-dimensional Bessel process, using space and time changes related to the Ray-Knight theorems on local times, in the manner of Jeulin [Lect. Notes Math., vol. 1118, Springer, Berlin, 1985] and Biane-Yor [Bull. Sci. Math. 2ème Sér. 111 (1987) 23-101].

Here, we characterize the law of a triplet linked to the perturbed Brownian motion which naturally arises in [Ph. Carmona et al., Stochastic Proc. Appl. 7 (1999) 323-333], and we point out its relations with Bessel processes of several dimensions.

The results provide some new understanding of the generalizations of Lévy's arc sine law for perturbed Brownian motions previously obtained by the second author.

(C) 2004 Elsevier SAS. All rights reserved.
\end{abstract}

\section{Résumé}

La décomposition trajectorielle de D. Williams' et le théorème de représentation de Pitman pour le processus de Bessel de dimension 3 sont l'expression de relations profondes entre le mouvement brownien réfléchi et le processus de Bessel de dimension 3.

Dans [Ph. Carmona et al., Stochastic Process. Appl. 7 (1999) 323-333], les résultats présentés permettent de mieux relier le mouvement brownien réfléchi et le processus de Bessel de dimension 2, en utilisant, entre autres, des changements de temps liés aux théorèmes de Ray-Knight sur les temps locaux, comme dans Jeulin [Lect. Notes Math., vol. 1118, Springer, Berlin, 1985] et Biane-Yor [Bull. Sci. Math. 2ème Sér. 111 (1987) 23-101].

Dans le présent article, nous caractérisons la loi d'un triplet lié au mouvement brownien perturbé qui apparaît naturellement dans [Ph. Carmona et al., Stochastic Proc. Appl. 7 (1999) 323-333], et nous mettons en évidence ses relations avec des processus de Bessel de plusieurs dimensions.

\footnotetext{
* Corresponding author.

E-mail addresses: philippe.carmona@math.univ-nantes.fr (Ph. Carmona), fpe@ ccr.jussieu.fr (F. Petit).
} 
Les résultats permettent d'améliorer notre compréhension de généralisations de la loi de l'arcsinus pour les mouvements browniens perturbés obtenues précédemment par le second auteur.

(c) 2004 Elsevier SAS. All rights reserved.

MSC: primary: 60J55, 60J60, 60J65

Keywords: Reflecting Brownian motion; Bessel processes; Ray-Knight theorems; Generalized arc-sine laws

\section{Introduction and main results}

1.1. The main question addressed in this paper is to determine the trivariate law of the random vector $\left(S_{t}^{Y}, l_{t}^{0}(Y), Y_{t}\right)$, for fixed $t>0$, associated with some remarkable semi-martingales $\left(Y_{t} ; t \geqslant 0\right)$, with $S_{t}^{Y} \stackrel{\text { def }}{=} \sup _{s \leqslant t} Y_{S}$, and $l_{t}^{0}(Y)$ the local time of $Y$ at level 0 , up to time $t$. Even for $Y_{t}=\beta_{t}+c t, t \geqslant 0$, a Brownian motion with drift $c$, this problem is not so simple to solve; see [24], for a solution in this case and some applications to the computation of the price of some exotic financial options.

As a second case of interest, we consider the $\mu$-perturbed Brownian motion $X^{\mu} \stackrel{\text { def }}{=}|B|-\mu l^{0}(B)$, where $\mu>0$ and $l_{t}=l_{t}^{0}(B)$ is the local time at 0 of $B$, to be precise: $l_{t}=\lim _{\varepsilon \rightarrow 0} \frac{1}{2 \varepsilon} \int_{0}^{t} d s 1_{\left|B_{s}\right| \leqslant \varepsilon}$. Thanks to Paul Lévy's identity,

$$
\left(\left|B_{t}\right|, l_{t}^{0}(B) ; t \geqslant 0\right) \stackrel{\text { law }}{=}\left(S_{t}^{B}-B_{t}, S_{t}^{B} ; t \geqslant 0\right),
$$

the $\mu$-perturbed Brownian motion may also be defined as:

$$
X_{t}^{\mu}=(1-\mu) S_{t}^{B}-B_{t},
$$

and it is not difficult to see that $Y=-X^{\mu}$ satisfies the equation

$$
Y_{t}=B_{t}+\left(1-\frac{1}{\mu}\right) S_{t}^{Y}
$$

In that second case, the computation of the joint law of $\left(S_{t}^{Y}, l_{t}^{0}(Y), Y_{t}\right)$ is much more complicated than for the first case (see [9], where this problem is considered but not solved).

As a third case study, we introduce, for $\delta>1$, the process $\rho^{(\delta)}$, defined as the unique adapted solution, taking values in $\mathbb{R}_{+}$, of the following equation (see e.g. [37] and [11]):

$$
\rho_{t}^{(\delta)}=\beta_{t}+(2-\delta) S_{t}^{(\delta)}+L_{t}^{(\delta)},
$$

where $S_{t}^{(\delta)}=\sup _{s \leqslant t} \rho_{s}^{(\delta)}$, and $l_{t}^{(\delta)}=2 L_{t}^{(\delta)}$ is the local time of $\rho^{(\delta)}$ at level 0 , and $\left(\beta_{t}\right)_{t \geqslant 0}$ is a Brownian motion starting from 0 .

When $\delta=2, \rho^{(\delta)}$ is a reflected Brownian motion $\left(\rho^{(2)}=|B|\right)$, and simple results are known about the joint law of the associated triplet, at least when considered at an independent exponential time (see Subsection 5.3; see also [45]). In the case $\delta \neq 2$, the process $\rho^{(\delta)}$ behaves like a reflected Brownian motion, except when it reaches a new maximum. Hence, it may be called the $(2-\delta)$-perturbed reflected Brownian motion. It appears in particular in the study of windings of planar Brownian motion [2,3,31] and is linked to the $\mu$-perturbed Brownian motion as follows. Let $\mu \stackrel{\text { def }}{=} \frac{1}{\delta-1}>0$, and consider the perturbed Brownian motion $X^{\mu} \stackrel{\text { def }}{=}|B|-\mu l^{0}(B)$. We denote by $A_{t}^{ \pm, \mu}=\int_{0}^{t} 1_{ \pm\left(\left|B_{s}\right|-\mu l_{s}^{0}(B)\right) \geqslant 0} d s$ the positive and negative times spent by $X^{\mu}$ before time $t$, and by $\left(\alpha_{t}^{ \pm, \mu}\right)$ their right continuous inverses. Then, there exists a standard Brownian motion $\beta$ such that (see e.g. [37], Part I, §5, p. 28, identities (37) and (39)):

$$
X_{\alpha_{t}^{-, \mu}}^{\mu /-}=\beta_{t}+\frac{\mu-1}{\mu} \sup _{s \leqslant t} X_{\alpha_{s}^{-, \mu}}^{\mu /-}+\frac{1}{2} l_{\alpha_{t}^{-, \mu}}^{0}\left(X^{\mu}\right),
$$


where $X_{s}^{\mu /-}=\sup \left(-X_{s}^{\mu}, 0\right)$ denotes the negative part of $X_{s}^{\mu}$. Thus, one recovers Eq. (1.3) for $\rho^{(\delta)}$, where:

$$
\rho_{t}^{(\delta)}=X_{\alpha_{t}^{-, \mu}}^{\mu /-}, \quad S_{t}^{(\delta)}=\mu l_{\alpha_{t}^{-, \mu}}^{0}(B), \quad \text { and } \quad L_{t}^{(\delta)}=\frac{1}{2} l_{\alpha_{t}^{-, \mu}}^{0}\left(X^{\mu}\right) .
$$

For more details on perturbed Brownian motions, perturbed reflected Brownian motions and perturbed Bessel processes, see e.g. [18-20,36,39].

We became all the more motivated by our initial question when looking again at two results we obtained previously (Theorems 1.1 and 1.2 below), while seeking for a better understanding of why certain functionals of the so-called $\mu$-perturbed Brownian motion $X^{\mu}$ are beta-distributed. These theorems exhibit some relationship between the reflected Brownian motion and the two-dimensional Bessel process, and, more generally, perturbed reflected Brownian motions and Bessel processes.

Theorem 1.1 [8]. Let $\rho_{t} \stackrel{\text { def }}{=}\left|B_{t}\right|$ be a reflected Brownian motion, $S_{t} \stackrel{\text { def }}{=} \sup _{s \leqslant t} \rho_{s}$ its supremum, $l_{t} \stackrel{\text { def }}{=} l_{t}^{0}(B)$ the local time of a standard Brownian motion B. For every fixed $t>0$, the identity in law:

$$
\left(S_{t}, S_{t}-\rho_{t}+l_{t}\right) \stackrel{\text { law }}{=}\left(\frac{1}{2} \int_{0}^{t} \frac{d s}{R_{s}^{(2)}}, R_{t}^{(2)}\right)
$$

holds, where $\left(R_{s}^{(2)} ; s \geqslant 0\right)$ denotes a two-dimensional Bessel process, starting from 0.

Warning. We immediately indicate that the identity in law (1.4) cannot hold between the two processes $\left(R_{t}^{(2)}, t \geqslant 0\right)$, and $\left(S_{t}-\rho_{t}+l_{t}, t \geqslant 0\right)$, since, as is well-known, the first is recurrent, while the second satisfies $S_{t}-\rho_{t}+l_{t} \geqslant l_{t}$, hence it is transient. Likewise, the left hand sides of both 2-dimensional processes $\left(S_{t} ; t \geqslant 0\right)$ and $\left(\frac{1}{2} \int_{0}^{t} \frac{d s}{R_{s}^{(2)}} ; t \geqslant 0\right)$ cannot be equal in law as processes, since the first increasing process is singular, and the second is absolutely continuous.

Theorem 1.2 [8, Theorem 3]. For every fixed $t>0$, we have:

$$
\left(S_{t}^{(\delta)}, S_{t}^{(\delta)}-\rho_{t}^{(\delta)}+L_{t}^{(\delta)}\right) \stackrel{\text { law }}{=}\left(\frac{1}{2} \int_{0}^{t} \frac{d s}{R_{s}^{(\delta)}}, R_{t}^{(\delta)}\right),
$$

where $R^{(\delta)}$ is a $\delta$-dimensional Bessel process issued from $0(\delta>1)$.

\subsection{Notations}

From now on, we shall work with a parameter $\delta>1$. Since Bessel processes of various dimensions play an important role in this paper, we shall use the notation $\mathbb{P}^{(\delta)}$ or $\mathbb{E}^{(\delta)}$ for the law (or the expectation) of $\left(R_{s}\right)_{s} \geqslant 0$, a Bessel process of dimension $\delta$, starting from 0 , also denoted $\left(R_{S}^{(\delta)}\right)_{s \geqslant 0}$. We also write $\tilde{H}_{t} \stackrel{\text { def }}{=} \frac{1}{2} \int_{0}^{t} \frac{d s}{R_{s}}$.

Concerning various local times of semi-martingales $\left(Y_{t}\right)$ of the form $Y_{t}=B_{t}+A_{t}$, where $B$ is a Brownian motion, we take unless otherwise indicated Meyer's convention: $l_{t}^{a}(Y)=\lim _{\varepsilon \rightarrow 0} \frac{1}{\varepsilon} \int_{0}^{t} d s 1_{0 \leqslant Y_{s}-a \leqslant \varepsilon}$.

We use the notations $Z_{t}^{(\delta)} \stackrel{\text { def }}{=} S_{t}^{(\delta)}-\rho_{t}^{(\delta)}+L_{t}^{(\delta)}$ and $\xi_{t}^{(\delta)} \stackrel{\text { def }}{=}\left(S_{t}^{(\delta)}, Z_{t}^{(\delta)}, L_{t}^{(\delta)}\right)$. Notice that $\xi^{(\delta)}$ inherits the scaling property from the driving Brownian motion $\beta$ in (1.3).

Furthermore, $G_{a}$ denotes a gamma variable of parameter $a$, and $G_{a, b}$ a beta variable of parameters $a$ and $b$ :

$$
\mathbb{P}\left[G_{a} \in d x\right]=\frac{1}{\Gamma(a)} 1_{x>0} x^{a-1} e^{-x} d x,
$$




$$
\mathbb{P}\left[G_{a, b} \in d x\right]=\frac{1}{B(a, b)} 1_{0<x<1} x^{a-1}(1-x)^{b-1} d x .
$$

In particular, $G_{1}$ is an exponential variable with mean 1 also denoted sometimes by $\mathbf{e}$, and $G_{1,1}$ is a uniform variable on $[0,1]$, also denoted by $U$ or $V$.

We use the French abbreviations sh, ch, th and coth for hyperbolic functions.

Furthermore, at the end of this paper, one will find a table which summarizes the different processes and corresponding notations we use, a table to which the reader could refer all along if necessary.

\subsection{Main results}

Theorem 1.2 naturally prompted us to describe the trivariate law of $\left(S_{t}^{(\delta)}, \rho_{t}^{(\delta)}, L_{t}^{(\delta)}\right)$; the computation of this law and its links with Bessel processes is one of the main results of the present paper. Various interesting features of this law are discussed in detail in Section 5. For the moment, we present the following descriptions:

Theorem 1.3. Let $T$ be an exponential variable, with mean 2 , independent of $\rho^{(\delta)}$. The joint law of $\left(S_{T}^{(\delta)}, \rho_{T}^{(\delta)}, L_{T}^{(\delta)}\right)$ is given by:

$$
\begin{aligned}
& \mathbb{P}\left(S_{T}^{(\delta)} \in d s, \rho_{T}^{(\delta)} \in d x, L_{T}^{(\delta)} \in d l\right) \\
& \quad=1_{0<x<s, l>0} \frac{e^{-l \operatorname{coth} s}}{\Gamma(\delta-1)}\left\{\frac{l^{\delta-1}}{(\operatorname{sh} s)^{\delta+1}} \operatorname{sh}(s-x)+(\delta-1) \frac{l^{\delta-2}}{(\operatorname{sh} s)^{\delta}} \operatorname{sh} x\right\} d s d x d l,
\end{aligned}
$$

which is the consequence of the following proposition and Eq. (5.2).

Proposition 1.4. Let $T$ be an independent exponential time of parameter $\frac{1}{2}$. Then, for every positive $\alpha, \beta$ and $\gamma$, one has:

$$
\begin{aligned}
\left(1-\beta^{2}\right)\left[\exp \left(-\alpha S_{T}^{(\delta)}-\beta \rho_{T}^{(\delta)}-\gamma L_{T}^{(\delta)}\right)\right]= & 1-\frac{\alpha+(2-\delta) \beta}{2} \mathbb{E}^{\delta}\left[\frac{1}{R_{T}} \exp \left(-(\alpha+\beta) \tilde{H}_{T}-\gamma R_{T}\right)\right] \\
& -(\delta-1) \frac{\beta+\gamma}{2} \mathbb{E}^{\delta+1}\left[\frac{1}{R_{T}} \exp \left(-\alpha \tilde{H}_{T}-\gamma R_{T}\right)\right] .
\end{aligned}
$$

Our study of the perturbed Brownian motion $\rho^{(\delta)}$ led us to new results about the perturbed Brownian motion $X^{\mu}$ (see Section A.2 for details).

Proposition 1.5. Let $T$ be an independent exponential time with parameter $\frac{1}{2}$ and $\mu>0$. Then, if $S_{t}^{\mu} \stackrel{\text { def }}{=} \sup _{0 \leqslant s \leqslant t} X_{s}^{\mu}$ and $I_{t}^{\mu} \stackrel{\text { def }}{=} \sup _{0 \leqslant s \leqslant t}\left(-X_{s}^{\mu}\right)$, the law of $\left(X_{T}^{\mu}, S_{T}^{\mu}, I_{T}^{\mu}\right)$ is determined by:

$$
\begin{aligned}
\mathbb{P} & {\left[S_{T}^{\mu} \in d s,\left(S_{T}^{\mu}+I_{T}^{\mu}\right) \in d y,\left(S_{T}^{\mu}-X_{T}^{\mu}\right) \in d x\right] } \\
& =\frac{(\operatorname{sh} s)^{\frac{1}{\mu}-1}}{\mu(\operatorname{sh} y)^{2+\frac{1}{\mu}}}\left\{\frac{\operatorname{sh} x \operatorname{sh}(y-s)}{\mu}+\operatorname{sh} s \operatorname{sh}(y-x)\right\} 1_{0<x<y} 1_{0<s<y} d s d y d x .
\end{aligned}
$$

More generally, we obtain the Fourier-Laplace transform (A.10) for the doubly-perturbed Brownian motion [9,11]. There had been an attempt in that direction in [9]. 


\subsection{The rest of this paper is organised as follows:}

- in Section 2, we explain how Theorem 1.2 is related to certain extensions of Lévy's arcsine and uniform laws, and we present our motivation for Theorem 1.3 and some interesting corollaries which we did not give in [8];

- in Section 3, we give a characterization of the law of the triplet $\xi_{t}^{(\delta)}$ as the solution of an integro-differential equation involving the laws of the right hand side of Eq. (1.5) for Bessel processes with dimensions $\delta$ and $\delta+1$

- the details of the proof of this characterization are given in Section 4;

- in Section 5, we use this characterization to obtain first the Laplace transform of $\xi_{T}^{(\delta)}$, where $T$ is an independent exponential variable, and then the law of $\xi_{T}^{(\delta)}$, which we already presented briefly in the above Theorem 1.3; we give some explicit expressions for the laws of the variables we study; then, we explain the result thanks to a four-dimensional identity in law, the proof of which involves Markovian processes and is postponed to Appendix A;

- finally, in this appendix, we develop the proofs of Proposition 5.11 and of Eq. (5.19) in Proposition 5.12, and we explain further how this work is related to the joint law of the doubly perturbed Brownian motion, its maximum and its minimum at an independent exponential time (Proposition 1.5).

\section{Our motivation for Theorems 1.2 and 1.3}

2.1. We now briefly explain how we found the relations (1.4) and (1.5) while seeking for some better understanding of certain extensions of Paul Lévy's arc sine and uniform laws (see the introduction of [8] for more details; see also $[10,51])$ :

$$
\int_{0}^{1} 1_{B_{s} \leqslant 0} d s \stackrel{\text { law }}{=} G_{\frac{1}{2}, \frac{1}{2}} \text { and } \int_{0}^{1} 1_{b_{s} \leqslant 0} d s \stackrel{\text { law }}{=} U,
$$

where $\left(b_{s} ; s \leqslant 1\right)$ denotes a standard Brownian bridge. These extensions, due to the second author (see [37], [38] and [50] p. 102, formula (8.6)) are as follows: for any $\mu>0$,

$$
A_{g}^{-, \mu} \stackrel{\text { law }}{=} G_{\frac{1}{2}, \frac{1}{2}+\frac{1}{2 \mu}}
$$

where $g=\sup \left\{t \leqslant 1 ; B_{t}=0\right\}, A_{t}^{ \pm, \mu}=\int_{0}^{t} 1_{ \pm\left(\left|B_{s}\right|-\mu l_{s}^{0}(B)\right) \geqslant 0} d s$. Then, denoting by $\left(\alpha_{t}^{-, \mu}\right)$ the right continuous inverse of $\left(A_{s}^{-, \mu}\right)$, we show that

$$
A_{g}^{-, \mu} \stackrel{\text { law }}{=} \frac{1}{1+\left(\frac{1}{2} l_{\alpha_{1}^{-, \mu}}^{\mu}+\left|B_{\alpha_{1}^{-, \mu}}\right|\right)^{2} \hat{T}},
$$

where $l^{\mu}=l^{0}\left(X^{\mu}\right)$ is the local time of $X^{\mu} \stackrel{\text { def }}{=}|B|-\mu l^{0}(B)$ at level 0 , and where $\hat{T}$ denotes the first hitting time of 1 by a Brownian motion $\hat{B}$, independent of the Brownian motion $B$.

2.2. Consequently, we were naturally interested in the law of the pair $\left(l_{\alpha_{1}^{-, \mu}}^{0}\left(X^{\mu}\right), B_{\alpha_{1}^{-, \mu}}\right)$. As we saw in the introduction, this pair may be represented in terms of the process $\rho^{(\delta)}$, so that

$$
Z_{t}^{(\delta)}=\frac{1}{2} l_{\alpha_{t}^{-, \mu}}^{0}\left(X^{\mu}\right)+\left|B_{\alpha_{t}^{-, \mu}}\right|=X_{\alpha_{t}^{-, \mu}}^{\mu}-\inf _{s \leqslant t} X_{\alpha_{s}^{-, \mu}}^{\mu}+\frac{1}{2} l_{\alpha_{t}^{-, \mu}}^{0}\left(X^{\mu}\right) .
$$


Now, Theorem 1.2 may be re-written as follows:

Theorem 2.1. For every fixed $t>0$ :

$$
\left(\mu l_{\alpha_{t}^{-, \mu}}^{0}(B), \frac{1}{2} l_{\alpha_{t}^{-, \mu}}^{0}\left(X^{\mu}\right)+\left|B_{\alpha_{t}^{-, \mu}}\right|\right) \stackrel{\text { law }}{=}\left(\frac{1}{2} \int_{0}^{t} \frac{d s}{R_{s}^{\left(1+\frac{1}{\mu}\right)}}, R_{t}^{\left(1+\frac{1}{\mu}\right)}\right) .
$$

2.3. Before going on, we now present some variants and corollaries of our Theorems 1.1 and 1.2. We find these to be interesting remarks; furthermore, they should provide examples of weak Bessel processes, that is processes $\left(Z_{t} ; t \geqslant 0\right)$ which are distributed as Bessel processes at any time $t$ but not as processes. (Likewise, weak Brownian motions are constructed in [23]; see [40]).

(1) Thanks to Lévy's identity (1.1), one deduces:

$$
\left(S_{t}^{|B|} ; l_{t}^{0}(B)-\left|B_{t}\right| ; t \geqslant 0\right) \stackrel{\text { law }}{=}\left(\sup _{s \leqslant t}\left(S_{s}^{B}-B_{s}\right), B_{t} ; t \geqslant 0\right) .
$$

In particular, the process $\left(\sup _{0<r<s<t}\left(B_{r}+B_{t}-B_{s}\right) ; t \geqslant 0\right)$ which appears in [34] is distributed as $\left(Z_{t}=\right.$ $\left.S_{t}^{|B|}+l_{t}^{0}(B)-\left|B_{t}\right| ; t \geqslant 0\right)$, that is, as a consequence of the preceding result, like the two-dimensional Bessel $R_{t}^{(2)}$ at any fixed time $t$. Moreover, the greatest downfall before $t$ of a standard Brownian motion, i.e. $\sup _{s \leqslant t}\left(S_{s}^{B}-B_{s}\right)$, is distributed as $\frac{1}{2} \int_{0}^{t} \frac{d s}{R_{s}^{(2)}}$. Changing $B$ in $-B$, we recognize the score $\xi_{0}(t)$ of the Brownian motion $B$, which appears as the limit in distribution of the local score of a sequence of i.i.d. random variables (the local score is an important tool for DNA sequence analysis, see [17]). This variable also appears for instance in the study of the simple random walk in random environment (see [26] where it is denoted $W_{t}^{\sharp}$ with $W=-B$ ).

(2) Here, we fix $t>0$. $\left(B_{t}-B_{t-s}\right)_{0 \leqslant s \leqslant t}$ is a Brownian motion, the identity (2.3) may be rewritten as:

$$
\left(\sup _{s \leqslant t}\left(B_{s}-\inf _{s \leqslant v \leqslant t} B_{v}\right), B_{t}\right) \stackrel{\operatorname{law}}{=}\left(\frac{1}{2} \int_{0}^{t} \frac{d s}{R_{s}^{(2)}}, R_{t}^{(2)}-\frac{1}{2} \int_{0}^{t} \frac{d s}{R_{s}^{(2)}}\right) .
$$

(3) Thanks to the well-known Cameron-Martin relationship between Brownian motion and Brownian motion with drift, the result of Theorem 1.1 may be extended to some couples of diffusions, indexed by a parameter $c \in \mathbb{R}$.

On one hand, we consider the bang-bang diffusion $Y^{(c)}$, with parameter $c$, which is defined as the solution of (see [29], [1] and [28], Chapter VI.5):

$$
Y_{t}=B_{t}-c \int_{0}^{t} \operatorname{sgn}\left(Y_{s}\right) d s
$$

We recall the extension of Lévy's representation of reflected Brownian motion by Fitzsimmons [22], Graversen and Shiryaev [25], Cherny and Shiryaev [13]:

$$
\left(\left|Y_{t}^{(c)}\right|, \tilde{L}_{t}^{(c)} ; t \geqslant 0\right) \stackrel{\text { law }}{=}\left(\hat{S}_{t}^{(c)}-\hat{B}_{t}^{(c)}, \hat{S}_{t}^{(c)} ; t \geqslant 0\right),
$$

where, in order to avoid confusion with other processes, we have denoted:

$$
\hat{B}_{t}^{(c)}=B_{t}+c t, \quad \hat{S}_{t}^{(c)}=\sup _{s \leqslant t}\left(\hat{B}_{s}^{(c)}\right),
$$

and $\tilde{L}^{(c)}$ denotes the local time at 0 of $Y^{(c)}$. Of course, formula (2.5) immediately follows from Itô's formula and Skorokhod's lemma. 
On the other hand, we introduce the 2-dimensional Bessel process, $R^{(2, c)}$ with "naïve" drift $c$ (which plays some important role in [49]), as the solution of:

$$
R_{t}=B_{t}+c t+\frac{1}{2} \int_{0}^{t} \frac{d s}{R_{s}} .
$$

Then, Theorem 1.1 may be extended as follows:

Theorem 2.2. For every $c \in \mathbb{R}$ and $t>0$, the identity in law:

$$
\left(\tilde{S}_{t}^{(c)}, \tilde{S}_{t}^{(c)}-\tilde{\rho}_{t}^{(c)}+\tilde{L}_{t}^{(c)}\right) \stackrel{\text { law }}{=}\left(\frac{1}{2} \int_{0}^{t} \frac{d s}{R_{s}^{(2, c)}}, R_{t}^{(2, c)}\right)
$$

holds, where $\tilde{\rho}_{t}^{(c)}$ denotes the absolute value of the bang-bang diffusion $Y^{(c)}, \tilde{L}^{(c)}$ is the local time at 0 of $Y^{(c)}$, and $R^{(2, c)}$ is defined as above.

\section{A trivariate law}

3.1. The following theorem characterizes the law of $\xi_{t}^{(\delta)} \stackrel{\text { def }}{=}\left(S_{t}^{(\delta)}, Z_{t}^{(\delta)}, L_{t}^{(\delta)}\right)$. Note that, thanks to the scaling property: $\xi_{t}^{(\delta)} \stackrel{\text { law }}{=} \sqrt{t} \xi_{1}^{(\delta)}$.

Theorem 3.1. For every $f \in \mathcal{C}^{1,2,1}\left(\mathbb{R}_{+}^{3}, \mathbb{R}\right)$, one has, with the notations introduced in the introduction:

$$
\begin{aligned}
\mathbb{E}\left[f\left(\xi_{t}^{(\delta)}\right)\right]= & f(0)+\frac{1}{2} \mathbb{E}\left[\int_{0}^{t} \frac{\partial^{2} f}{\partial z^{2}}\left(\xi_{u}^{(\delta)}\right) d u\right]+\mathbb{E}^{(\delta)}\left[\int_{0}^{t}\left(\frac{\partial f}{\partial s}+(\delta-1) \frac{\partial f}{\partial z}\right)\left(\tilde{H}_{u}, R_{u}, R_{u}\right) d \tilde{H}_{u}\right] \\
& +(\delta-1) \mathbb{E}^{(\delta+1)}\left[\int_{0}^{t} \frac{\partial f}{\partial l}\left(\tilde{H}_{u}, \tilde{H}_{u}+R_{u}, R_{u}\right) d \tilde{H}_{u}\right] .
\end{aligned}
$$

Proof. The proof of Theorem 3.1 relies upon:

- the combination of Itô's formula together with the two following important lemmata, whose proofs are postponed to the next Section 4;

- the fact that $Z^{(\delta)}=L^{(\delta)}$ when $S^{(\delta)}$ increases, $Z^{(\delta)}=L^{(\delta)}+S^{(\delta)}$ when $L^{(\delta)}$ increases, and $Z^{(\delta)}=\beta+(\delta-1) S^{(\delta)}$ according to (1.3).

Lemma 3.2 [8, Proposition 9]. For every Borel positive function $f: \mathbb{R}_{+}^{3} \rightarrow \mathbb{R}_{+}$, we have, denoting $Z^{(\delta)} \stackrel{\text { def }}{=} S^{(\delta)}-$ $\rho^{(\delta)}+L^{(\delta)}$ :

$$
\mathbb{E}\left[\int_{0}^{\infty} d S_{s}^{(\delta)} f\left(s, S_{s}^{(\delta)}, L_{s}^{(\delta)}\right)\right]=\mathbb{E}^{(\delta)}\left[\int_{0}^{\infty} d \tilde{H}_{s} f\left(s, \tilde{H}_{s}, R_{s}\right)\right] .
$$


Lemma 3.3. For every Borel positive function $f: \mathbb{R}_{+}^{3} \rightarrow \mathbb{R}_{+}$, we have:

$$
\mathbb{E}\left[\int_{0}^{\infty} d L_{s}^{(\delta)} f\left(s, S_{s}^{(\delta)}, L_{s}^{(\delta)}\right)\right]=(\delta-1) \mathbb{E}^{(\delta+1)}\left[\int_{0}^{\infty} d \tilde{H}_{s} f\left(s, \tilde{H}_{s}, R_{s}\right)\right] .
$$

3.2. A proof of Theorem 1.2 now follows easily from Theorem 3.1. We take $f(s, z)=\phi(s) \exp (-b z)$ with $\phi \in \mathcal{C}^{1}\left(\mathbb{R}_{+}\right)$, and we define

$$
F_{t}^{(\delta)}(b)=\mathbb{E}^{(\delta)}\left[\int_{0}^{t}\left(\phi^{\prime}\left(\tilde{H}_{s}\right)-(\delta-1) b \phi\left(\tilde{H}_{s}\right)\right) \exp \left(-b R_{s}\right) d \tilde{H}_{s}\right] .
$$

We note that both functions $\Phi_{t}^{(\delta)}(b)=\mathbb{E}\left[\phi\left(S_{t}^{(\delta)}\right) \exp \left(-b Z_{t}^{(\delta)}\right)\right]$ and $\tilde{\Phi}_{t}^{(\delta)}(b)=\mathbb{E}^{(\delta)}\left[\phi\left(\tilde{H}_{t}\right) \exp \left(-b R_{t}\right)\right]$ satisfy the same following equation:

$$
\psi_{t}(b)=\phi(0)+\frac{b^{2}}{2} \int_{0}^{t} \psi_{s}(b) d s+F_{t}^{(\delta)}(b) .
$$

This is obtained, for $\Phi_{t}^{(\delta)}$ thanks to Theorem 3.1, and for $\tilde{\Phi}_{t}^{(\delta)}$ thanks to Itô's formula, since the Bessel process of dimension $\delta$ satisfies

$$
d R_{t}=d \gamma_{t}+(\delta-1) d \tilde{H}_{t},
$$

where $\gamma$ is a Brownian motion ([47], Chapter XI, Exercise 1.28, p. 451).

As a consequence of (3.4), we find:

$$
\psi_{t}(b)=\exp \left(\frac{b^{2}}{2} t\right)\left\{\phi(0)+\int_{0}^{t} \exp \left(-\frac{b^{2} s}{2}\right) d_{s}\left(F_{s}^{(\delta)}(b)\right)\right\} .
$$

In particular:

$$
\Phi_{t}^{(\delta)}(b)=\tilde{\Phi}_{t}^{(\delta)}(b)
$$

thus proving Theorem 1.2.

Remark 3.4. A variant of the above arguments shows that the law of $\xi_{1}^{(\delta)}$ is uniquely determined, via Eq. (3.1), in terms of the laws of $\left(\tilde{H}_{1}, R_{1}, \mathbb{P}^{(\delta)}\right)$ and $\left(\tilde{H}_{1}, R_{1}, \mathbb{P}^{(\delta+1)}\right)$, as asserted in Section 3.1 above.

\section{Proofs and amplifications of Lemmata 3.2 and 3.3}

The proofs rely upon some adequate extensions of the classical Ray-Knight theorems for Brownian local times, as well as on some time substitutions transforming squared Bessel processes into Bessel processes, and vice-versa. Here are the details. Other references on Ray-Knight theorems are [15,16,21,27,35].

4.1. We first recall the basic Ray-Knight theorems, which we present here (this is more convenient for our present purpose) for the local times of the reflecting Brownian motion $\left(\rho_{t}=\left|B_{t}\right| ; t \geqslant 0\right)$; we denote these local times as $\left(l_{t}^{x}(\rho) ; x \geqslant 0, t \geqslant 0\right)$; they are jointly continuous, and satisfy the density of occupation formula:

$$
\int_{0}^{t} f\left(\rho_{s}\right) d s=\int_{0}^{\infty} f(x) l_{t}^{x}(\rho) d x=\int_{0}^{\infty} f(x)\left(l_{t}^{x}(B)+l_{t}^{-x}(B)\right) d x,
$$


for every Borel function $f: \mathbb{R}_{+} \rightarrow \mathbb{R}_{+}$.

(RK1) Let $a>0$, and $T_{a}^{*} \stackrel{\text { def }}{=} \inf \left\{t ; \rho_{t}=a\right\}$. Then, one has:

$$
\left\{l_{T_{a}^{*}}^{a-x}(\rho) ; 0 \leqslant x \leqslant a\right\} \stackrel{\text { law }}{=}\left\{\left(R_{x}^{(2)}\right)^{2} ; 0 \leqslant x \leqslant a\right\},
$$

where $R^{(2)}$ is a Bessel process of dimension 2 starting at 0 .

(RK2) Let $l>0$, and $\tau_{l} \stackrel{\text { def }}{=} \inf \left\{t ; l_{t}^{0}(\rho)>l\right\}=\inf \left\{t ; l_{t}^{0}(B)>\frac{l}{2}\right\}$. Then, $\left\{l_{\tau_{l}}^{x}(\rho) ; x \geqslant 0\right\}$ is distributed as a squared Bessel process of dimension 0 starting at $l$.

4.2. Here are the promised extensions of (RK1) and (RK2) to the local times of $\rho^{(\delta)}$.

Theorem 4.1. Let $\delta>1$, and $\hat{\delta}=2(\delta-1)$, and $\rho^{(\delta)}$ denotes the process defined in (1.3). We denote by $\left(l_{t}^{x}\left(\rho^{(\delta)}\right) ; x \geqslant 0, t \geqslant 0\right)$ its jointly continuous family of local times. Then:

$(\mathrm{RK} 1)_{\delta}$ if $T_{a}^{(\delta)} \stackrel{\text { def }}{=} \inf \left\{t ; \rho_{t}^{(\delta)}=a\right\}$, the process $\left\{l_{T_{a}^{(\delta)}}^{a-x}\left(\rho^{(\delta)}\right) ; 0 \leqslant x \leqslant a\right\}$ is distributed as the square of a $\hat{\delta}$-dimensional Bessel process starting from 0 ;

$(\mathrm{RK} 2)_{\delta}$ if $\tau_{l}^{(\delta)} \stackrel{\text { def }}{=} \inf \left\{t ; l_{t}^{0}\left(\rho^{(\delta)}\right)>l\right\}$, then, $\left\{l_{\tau_{l}^{(\delta)}}^{x}\left(\rho^{(\delta)}\right) ; x \geqslant 0\right\}$ is distributed as the square of a Bessel process of dimension $2(2-\delta)$, starting at $l$, and absorbed when it reaches 0 .

Proof. The proof is based on the representation of the process $\left(\rho_{t}^{(\delta)}\right)_{t \geqslant 0}$, already explained in Section 2.2. Here, we drop the subscript $\mu$ to make notations clearer.

Denoting by $l_{t}^{a}\left(\rho^{(\delta)}\right)$ the local time of $\rho^{(\delta)}$ at time $t$ and level $a$, and by $T_{-1}(X)$ the first hitting time of -1 by $X$, we obtain:

$$
\begin{aligned}
& \left.T_{1}^{(\delta)}=\inf \left\{s ; X_{\alpha_{s}^{-}}^{-}=1\right\}=A_{\inf \left\{s ; X_{\alpha_{s}^{-}}^{-}\right.}^{-}=1\right\} \\
& \forall a>0, \quad l_{T_{1}^{(\delta)}}^{a}\left(\rho^{(\delta)}\right)=A_{T_{-1}(X)}^{-a}, \\
& \forall(X),
\end{aligned}
$$

and

$$
\frac{1}{2} l_{T_{1}^{(\delta)}}^{(\delta)}=L_{T_{1}^{(\delta)}}^{(\delta)}=\frac{1}{2} l_{T_{-1}(X)}^{0}(X)
$$

Thanks to [30,41] (Corollary 2.2), [4] and [7] (Corollary 3.4.1ii) pages 15 and 16), we know that the local time process $\left(l_{T_{-1}(X)}^{a-1}(X) ; 0 \leqslant a \leqslant 1\right)$ is distributed as a squared Bessel process starting at 0 and of dimension $\frac{2}{\mu}=2(\delta-1)=\hat{\delta}$. This gives the first desired result.

With the same arguments, we have $l_{\tau_{l}^{(\delta)}}^{x}\left(\rho^{(\delta)}\right)=l_{\tau_{l}^{\mu}}^{-x}(X)$ for every $x \geqslant 0$, and Theorem 3.3 of [7] gives the second desired result.

4.3. We now prove Lemma 3.3. In order to vary our arguments from the proof of Lemma 3.2 given in [8], we shall present a general identity involving essentially the local times of $\rho^{(\delta)}$, up to $\tau_{l}^{(\delta)}$.

Proposition 4.2. For every positive functional $\Phi$ on $\mathcal{C}\left(\mathbb{R}_{+}, \mathbb{R}_{+}\right)$, one has:

$$
\mathbb{E}\left[\int_{0}^{\infty} d L_{s}^{(\delta)} \Phi\left(l_{s}^{S_{s}^{(\delta)}-x}\left(\rho^{(\delta)}\right) ; x \leqslant S_{s}^{(\delta)}\right)\right]=(\delta-1) \mathbb{E}^{(2 \delta)}\left[\int_{0}^{\infty} d t \Phi\left(R_{x}^{2} ; x \leqslant t\right)\right] .
$$


Proof. We first make the obvious time-change on the left-hand side of (4.1) which we denote by $I$. Thus, this term takes the form (we drop the superscript $\delta$ here):

$$
2 I=\left[\int_{0}^{\infty} d l \Phi\left(l_{\tau_{l}}^{S_{\tau_{l}}-x} ; x \leqslant S_{\tau_{l}}\right)\right]=\int_{0}^{\infty} \mathbb{Q}_{l}^{(4-2 \delta)}\left[\Phi\left(V_{T_{0}-x} ; x \leqslant T_{0}\right)\right] d l
$$

thanks to the second part of Theorem 4.1, and where $\mathbb{Q}_{l}^{d}$ is the law of the square of a $d$-dimensional Bessel process $V$, starting at $l$, killed at $T_{0}=\inf \left\{y ; V_{y}=0\right\}$.

Using D. Williams' time reversal theorem (cf. [47], Chapter IX, exercise 1.23, p. 451), the previous expression is equal to

$$
2 I=\int_{0}^{\infty} \mathbb{Q}_{0}^{(2 \delta)}\left[\Phi\left(V_{x} ; x \leqslant \Lambda_{l}\right)\right] d l
$$

where $\Lambda_{l}=\sup \left\{s ; V_{s}=l\right\}$. Using Fubini's theorem, and the inverse $\left\{J_{s}=\inf _{u \geqslant s} V_{u} ; s \geqslant 0\right\}$ of $\left\{\Lambda_{l} ; l \geqslant 0\right\}$, we obtain:

$$
2 I=\mathbb{Q}_{0}^{(2 \delta)}\left[\int_{0}^{\infty} \Phi\left(V_{x} ; x \leqslant s\right) d J_{s}\right],
$$

and from [52], Chapter XII, pp. 46-47 (see also [46,48]), we conclude

$$
I=(\delta-1) \mathbb{Q}_{0}^{(2 \delta)}\left[\int_{0}^{\infty} \Phi\left(V_{x} ; x \leqslant s\right) d s\right] .
$$

End of the proof of Lemma 3.3. From Proposition 4.2, we deduce:

$$
\mathbb{E}\left[\int_{0}^{\infty} d L_{s}^{(\delta)} f\left(s, S_{s}^{(\delta)}, L_{s}^{(\delta)}\right)\right]=(\delta-1) \mathbb{E}^{(2 \delta)}\left[\int_{0}^{\infty} d l f\left(\int_{0}^{l} R_{x}^{2} d x, l, \frac{1}{2} R_{l}^{2}\right)\right] .
$$

Then we use the time-substitution [47, Chapter XI, Proposition 1.11, p. 416] (see also [5]) transforming the square of the $2 \delta$-dimensional Bessel process $\left(R_{x}^{2} ; x \geqslant 0\right)$ into a $\delta^{\prime}$-dimensional Bessel process with $2 \delta=2\left(\delta^{\prime}-1\right)$, i.e. $\delta^{\prime}=1+\delta$ :

$$
\begin{aligned}
\mathbb{E}\left[\int_{0}^{\infty} d L_{s}^{(\delta)} f\left(s, S_{s}^{(\delta)}, L_{s}^{(\delta)}\right)\right] & =(\delta-1) \mathbb{E}^{(\delta+1)}\left[\int_{0}^{\infty} f\left(4 s, H_{s}, 2 R_{S}\right) d H_{s}\right] \\
& =(\delta-1) \mathbb{E}^{(\delta+1)}\left[\int_{0}^{\infty} f\left(t, \tilde{H}_{t}, R_{t}\right) d \tilde{H}_{t}\right] \text { by scaling. }
\end{aligned}
$$

4.4. The following analogue of Proposition 4.2 is an immediate consequence of $(R K 1)_{\delta}$ (see Theorem 4.1 above).

Proposition 4.3. For every positive functional $\Phi$ on $\mathcal{C}\left(\mathbb{R}_{+}, \mathbb{R}_{+}\right)$, one has:

$$
\mathbb{E}\left[\int_{0}^{\infty} d S_{u}^{(\delta)} \Phi\left(l_{u}^{S_{u}^{(\delta)}-x}\left(\rho^{(\delta)}\right) ; x \leqslant S_{u}^{(\delta)}\right)\right]=(\delta-1) \mathbb{E}^{2(\delta-1)}\left[\int_{0}^{\infty} d s \Phi\left(R_{x}^{2} ; x \leqslant s\right)\right] .
$$




\section{The law of the triplet $\xi_{T}^{(\delta)}=\left(S_{T}^{(\delta)}, Z_{T}^{(\delta)}, L_{T}^{(\delta)}\right)$, at an independent exponential time $T$}

5.1. We now explain, with the help of Theorem 3.1, how we deduce the law of $\xi_{T}^{(\delta)}$, where $T$ is an independent exponential time, which, by scaling, may be assumed to have parameter $\frac{1}{2}$.

Proposition 5.1. Let $T$ an independent exponential time of parameter $\frac{1}{2}$. Then, for every positive $\alpha, \beta$ and $\gamma$, one has:

$$
\begin{aligned}
\left(1-\beta^{2}\right) \mathbb{E}\left[\exp \left(-\alpha S_{T}^{(\delta)}-\beta \rho_{T}^{(\delta)}-\gamma L_{T}^{(\delta)}\right)\right] \\
=1-\frac{\alpha+(2-\delta) \beta}{2} \mathbb{E}^{\delta}\left[\frac{1}{R_{T}} \exp \left(-(\alpha+\beta) \tilde{H}_{T}-\gamma R_{T}\right)\right]-(\delta-1) \frac{\beta+\gamma}{2} \mathbb{E}^{\delta+1}\left[\frac{1}{R_{T}} \exp \left(-\alpha \tilde{H}_{T}-\gamma R_{T}\right)\right] \\
=1-B\left(\frac{1}{2}, \frac{\delta-1}{2}\right) \frac{\alpha+(2-\delta) \beta}{2} \mathbb{E}\left[\exp \left(-\frac{\alpha+\beta}{2} A_{\delta-1}-\gamma B_{\delta-1}\right)\right] \\
\quad-(\delta-1) B\left(\frac{1}{2}, \frac{\delta}{2}\right) \frac{\beta+\gamma}{2} \mathbb{E}\left[\exp \left(-\frac{\alpha}{2} A_{\delta}-\gamma B_{\delta}\right)\right]
\end{aligned}
$$

where the pair $\left(A_{2 d}, B_{2 d}\right)$ is distributed as $\left(\left|\ln \frac{G_{d}}{G_{d}^{\prime}}\right|,\left|G_{d}-G_{d}^{\prime}\right|\right), G_{d}$ and $G_{d}^{\prime}$ being two independent gamma variables of parameter $d$.

Proof. We prove the first equality in Proposition 5.1 applying Theorem 3.1 to $f(s, z, l)=\exp (-a s-b z-c l)$. To obtain the second equality, we use the following representation (see [43]):

$$
\mathbb{E}\left[\exp \left(-\frac{\lambda}{2} A_{2 d}-\gamma B_{2 d}\right)\right]=\frac{\Gamma\left(d+\frac{1}{2}\right)}{\Gamma(d) \Gamma\left(\frac{1}{2}\right)} \mathbb{E}^{2 d+1}\left[\frac{1}{R_{T}} \exp \left(-\lambda \tilde{H}_{T}-\gamma R_{T}\right)\right] .
$$

Remark 5.2. As a consequence of Theorem 1.2 and of the definition of $\left(A_{\delta-1}, B_{\delta-1}\right)$, we obtain some identities between the gamma and beta variables which appear in these identities in law. We may show they are in fact the consequence of the following identity, in the particular case $a=\frac{\delta-1}{2}$ :

$$
G_{a, a} \wedge\left(1-G_{a, a}\right) \stackrel{\operatorname{law}}{=} \frac{1-\sqrt{G_{\frac{1}{2}, a}}}{2} .
$$

For more details about the pair $\left(A_{2 d}, B_{2 d}\right)$, see [43].

In this section, we also note that the joint law of either side of (1.5) may be characterized explicitly as follows (see [43]):

Proposition 5.3. Let $\left(U^{(\delta)}, V^{(\delta)}\right)$ be a pair of random variables which follows the common law of either side of (1.5) for $t=1$. Then for every $\alpha \geqslant 0$ and $\beta \geqslant 0$, one has:

$$
\mathbb{E}\left[\left(U^{(\delta)}\right)^{\alpha}\left(V^{(\delta)}\right)^{\beta}\right]=\frac{\Gamma(\delta+\beta)}{2^{\frac{\alpha+\beta}{2}} \Gamma\left(1+\frac{\alpha+\beta}{2}\right) \Gamma(\delta-1)} \int_{0}^{\infty} z^{\alpha} \frac{(\operatorname{sh} z)^{\beta+1}}{(\operatorname{ch} z)^{\delta+\beta}} d z .
$$

In particular, $\mathbb{E}\left[\left(V^{(\delta)}\right)^{\beta}\right]=2^{\beta / 2} \Gamma\left(\frac{\delta+\beta}{2}\right) / \Gamma\left(\frac{\delta}{2}\right)$.

Then, inverting the Laplace transform written in (5.1) with usual computations and the help of Lebedev [32], we obtain the trivariate law of $\left(S_{T}^{(\delta)}, \rho_{T}^{(\delta)}, L_{T}^{(\delta)}\right)$ in the form presented in Theorem 1.3. 
5.2. Now, we shall discuss in detail a number of features of this trivariate law.

Theorem 5.4. The joint law of $\left(S_{T}^{(\delta)}, \rho_{T}^{(\delta)}, L_{T}^{(\delta)}\right)$ may be described as follows:

(i)

$$
\mathbb{P}\left(S_{T}^{(\delta)} \in d s, \rho_{T}^{(\delta)} \in d x\right)=(\delta-1) 1_{0<x<s} \frac{\operatorname{ch} x}{(\operatorname{ch} s)^{\delta}} d s d x,
$$

i.e. denoting by $U$ and $V$ two independent variables uniformly distributed on $[0,1]$,

$$
\left(\frac{1}{\operatorname{ch}\left(S_{T}^{(\delta)}\right)}, \frac{\operatorname{sh}\left(\rho_{T}^{(\delta)}\right)}{\operatorname{sh}\left(S_{T}^{(\delta)}\right)}\right) \stackrel{\text { law }}{=}\left(U^{\frac{1}{\delta-1}}, V\right) .
$$

(ii)

$$
\mathbb{P}\left[L_{T}^{(\delta)} \operatorname{coth}\left(S_{T}^{(\delta)}\right) \in d l / S_{T}^{(\delta)}=s, \rho_{T}^{(\delta)}=\rho\right]=\frac{\operatorname{th} \rho}{\operatorname{th} s} \mathbb{P}\left[G_{\delta-1} \in d l\right]+\left(1-\frac{\operatorname{th} \rho}{\operatorname{th} s}\right) \mathbb{P}\left[G_{\delta} \in d l\right] .
$$

Thanks to the scaling property, we deduce [32]:

\section{Corollary 5.5.}

$$
\begin{aligned}
\mathbb{P}\left[S_{1}^{(\delta)} \in d s, \rho_{1}^{(\delta)} \in d x\right]= & 2^{\delta-\frac{1}{2}} \sum_{k \geqslant 0} \frac{(-1)^{k} \Gamma(k+\delta)}{\sqrt{\pi} \Gamma(\delta-1) k !}\left\{[(2 k+\delta) s-x] e^{-\frac{[(2 k+\delta) s-x]^{2}}{2}}\right. \\
& \left.+[(2 k+\delta) s+x] e^{-\frac{[(2 k+\delta) s+x]^{2}}{2}}\right\} 1_{0<x<s} d x d s .
\end{aligned}
$$

For convenience, we now present the three 1-dimensional marginals.

\section{Corollary 5.6.}

$$
\left.S_{T}^{(\delta)} \stackrel{\operatorname{law}}{=} \operatorname{Argch}\left(1 / U^{\frac{1}{\delta-1}}\right]\right),
$$

i.e.

$$
\begin{aligned}
& \mathbb{P}\left(S_{T}^{(\delta)} \in d s\right)=(\delta-1) \frac{\operatorname{sh} s}{(\operatorname{ch} s)^{\delta}} 1_{s>0} d s . \\
& L_{T}^{(\delta)} \stackrel{\text { law }}{=} R_{T}^{(\delta-1)}, \\
& \rho_{T}^{(\delta)} \stackrel{\text { law }}{=} \operatorname{Argsh}\left(V \sqrt{\mathbf{e} / G_{\frac{\delta-1}{2}}}\right),
\end{aligned}
$$

where $V$, $\mathbf{e}$ and $G_{\frac{\delta-1}{2}}$ are independent variables, that is

$$
\mathbb{P}\left(\rho_{T}^{(\delta)} \in d x\right)=\frac{2^{\delta}(\delta-1)}{\delta} e^{-\delta x}(\operatorname{ch} x)_{2} F_{1}\left(\delta, \frac{\delta}{2} ; 1+\frac{\delta}{2} ;-e^{-2 x}\right) 1_{x>0} d x,
$$

where ${ }_{2} F_{1}(a, b ; c ; z)$ denotes the hypergeometric function (see [32]).

Consequently, we obtain:

$$
\begin{aligned}
& L_{1}^{(\delta)} \stackrel{\text { law }}{=} R_{1}^{(\delta-1)} \stackrel{\text { law }}{=} \sqrt{2 G_{\frac{\delta-1}{2}}}, \\
& Z_{1}^{(\delta)} \stackrel{\text { law }}{=} R_{1}^{(\delta)} \stackrel{\text { law }}{=} \sqrt{2 G_{\frac{\delta}{2}}},
\end{aligned}
$$


if $\delta \neq 2$,

$$
\begin{aligned}
\mathbb{P} & {\left[\rho_{1}^{(\delta)} \in d x\right] } \\
& =\frac{2^{\delta-\frac{1}{2}}}{\Gamma(\delta-2) \sqrt{\pi}} \sum_{k \geqslant 0}(-1)^{k} \frac{(2 k+\delta-1) \Gamma(k+\delta-1)}{k !(2 k+\delta)(2 k+\delta-2)} \exp \left(-\frac{(2 k+\delta-1)^{2} x^{2}}{2}\right) 1_{x>0} d x . \\
\mathbb{P} & {\left[S_{1}^{(\delta)} \in d s\right] } \\
& =\frac{2^{\delta-\frac{1}{2}}}{\sqrt{\pi}} \sum_{k \geqslant 0}(-1)^{k} \frac{(2 k+\delta-1) \Gamma(k+\delta-1)}{k ! \Gamma(\delta-1)} \exp \left(-\frac{(2 k+\delta-1)^{2} s^{2}}{2}\right) 1_{s>0} d s .
\end{aligned}
$$

5.3. In the case $\delta=2$, we recover known results (see e.g. [6], p. 333), and we note that these results may be deduced from the following four-variate law, which is obtained using, by now, classical excursion theoretic arguments.

We write $S_{T}=m_{T} \vee M_{T}$, where $m_{T}=\sup _{u \leqslant g_{T}} \rho_{u}$, and $M_{T}=\sup _{g_{T} \leqslant u \leqslant T} \rho_{u}$. Then the pairs $\left(m_{T}, L_{T}\right)$ and $\left(M_{T}, \rho_{T}\right)$ are independent. Furthermore:

Lemma 5.7. The joint laws of $\left(m_{T}, L_{T}\right)$ and $\left(M_{T}, \rho_{T}\right)$ are given by:

(i) $\mathbb{P}\left(m_{T} \leqslant u, L_{T} \in d l\right)=e^{-l \operatorname{coth} u} 1_{l>0, u>0} d l$;

(ii) $\mathbb{P}\left(M_{T} \in d y, \rho_{T} \in d x\right)=\frac{\operatorname{sh} x}{(\operatorname{sh} y)^{2}} d x d y 1_{0<x \leqslant y}$.

See also, e.g. [47], Chapter XII, Exercise 4.24, p. 510 (or [6]). From Lemma 5.7, we understand better formula (5.5) in this case:

Corollary 5.8. For any positive measurable function $f$, we have:

$$
\mathbb{E}\left[f\left(L_{T} \operatorname{coth}\left(S_{T}\right)\right) 1_{m_{T}<M_{T}} / S_{T}, \rho_{T}\right]=\frac{\operatorname{th}\left(\rho_{T}\right)}{\operatorname{th}\left(S_{T}\right)} \mathbb{E}\left[f\left(G_{1}\right)\right]
$$

and

$$
\mathbb{E}\left[f\left(L_{T} \operatorname{coth}\left(S_{T}\right)\right) 1_{M_{T} \leqslant m_{T}} / S_{T}, \rho_{T}\right]=\left(1-\frac{\operatorname{th}\left(\rho_{T}\right)}{\operatorname{th}\left(S_{T}\right)}\right) \mathbb{E}\left[f\left(G_{2}\right)\right] .
$$

Remark 5.9. Note in particular that the variable $L_{T} \operatorname{coth}\left(S_{T}\right)$ and the set $\left\{m_{T}<M_{T}\right\}$ are not conditionally independent given the pair $\left(S_{T}, \rho_{T}\right)$.

5.4. Now, we explain how Corollary 5.8 extends to the general case $\delta>1$, and yields a better understanding of formula (5.5). Let us introduce some notations:

$$
g_{t}^{(\delta)}=\sup \left\{s \leqslant t ; \rho_{s}^{(\delta)}=0\right\}, \quad m_{t}^{(\delta)}=\sup _{s \leqslant g_{t}^{(\delta)}} \rho_{s}^{(\delta)}, \quad \text { and } \quad M_{t}^{(\delta)}=\sup _{g_{t}^{(\delta)} \leqslant s \leqslant t} \rho_{s}^{(\delta)},
$$

so that $S_{t}^{(\delta)}=\max \left(m_{t}^{(\delta)}, M_{t}^{(\delta)}\right)$.

Proposition 5.10. For any positive measurable function $f$, we have:

$$
\mathbb{E}\left[f\left(L_{T}^{(\delta)} \operatorname{coth}\left(S_{T}^{(\delta)}\right)\right) 1_{m_{T}^{(\delta)}<M_{T}^{(\delta)} / S_{T}^{(\delta)}}, \rho_{T}^{(\delta)}\right]=\frac{\operatorname{th}\left(\rho_{T}^{(\delta)}\right)}{\operatorname{th}\left(S_{T}^{(\delta)}\right)} \mathbb{E}\left[f\left(G_{\delta-1}\right)\right]
$$


and

$$
\mathbb{E}\left[f\left(L_{T}^{(\delta)} \operatorname{coth}\left(S_{T}^{(\delta)}\right)\right) 1_{M_{T}^{(\delta)} \leqslant m_{T}^{(\delta)}} / S_{T}^{(\delta)}, \rho_{T}^{(\delta)}\right]=\left(1-\frac{\operatorname{th}\left(\rho_{T}^{(\delta)}\right)}{\operatorname{th}\left(S_{T}^{(\delta)}\right)}\right) \mathbb{E}\left[f\left(G_{\delta}\right)\right] .
$$

Let us notice first, that, in terms of perturbed Brownian motion (see Section 2.2), we have:

$$
\begin{aligned}
& g_{t}^{(\delta)}=A^{-, \mu}\left(g_{\alpha_{t}^{-, \mu}}^{\mu}\right), \quad \text { where } g_{t}^{\mu}=\sup \left\{u \leqslant t ; X_{u}^{\mu}=0\right\}, \\
& \text { and } m_{t}^{(\delta)}=\mu l_{g_{\alpha_{t}^{-, \mu}}^{\mu}} .
\end{aligned}
$$

As $\left\{m_{T}^{(\delta)}<M_{T}^{(\delta)}\right\}=\left\{m_{T}^{(\delta)}<S_{T}^{(\delta)}\right\}$ and $\left\{M_{T}^{(\delta)} \leqslant m_{T}^{(\delta)}\right\}=\left\{S_{T}^{(\delta)}=m_{T}^{(\delta)}\right\}$, it is equivalent, but more convenient, to study the quadruplet $\left(S_{T}^{(\delta)}, m_{T}^{(\delta)}, L_{T}^{(\delta)}, \rho_{T}^{(\delta)}\right)$, so that we are led to express $\mathbb{E}\left[F\left(S_{T}^{(\delta)}\right) G\left(m_{T}^{(\delta)}\right) f\left(L_{T}^{(\delta)}\right) k\left(\rho_{T}^{(\delta)}\right)\right]$, for any 4-tuple $(F, G, f, k)$ of positive measurable functions.

Proposition 5.11. Let $F, G, f$ and $k$ be four positive measurable functions. Then, using the relation $\delta=1+\frac{1}{\mu}$, we have:

$$
\begin{aligned}
\mathbb{E} & {\left[F\left(S_{T}^{(\delta)}\right) G\left(m_{T}^{(\delta)}\right) f\left(L_{T}^{(\delta)}\right) k\left(\rho_{T}^{(\delta)}\right) 1_{m_{T}^{(\delta)}<S_{T}^{(\delta)}}\right] } \\
& =\int_{\mathbb{R}_{+}^{2}} \frac{f(u) u^{1 / \mu} e^{-u \operatorname{coth} z} G(z) z^{2} d u d z}{2 \Gamma\left(\frac{1}{\mu}\right)(\operatorname{sh} z)^{2+1 / \mu}} \mathbb{E}\left[\int_{0}^{T_{1}\left(X^{\mu}\right)} F\left[z\left(1+\mu l_{x}^{0}(B)\right)\right] k\left[z\left(1-X_{x}^{\mu}\right)\right] e^{-z^{2} x / 2} d x\right]
\end{aligned}
$$

and

$$
\begin{aligned}
\mathbb{E} & {\left[F\left(S_{T}^{(\delta)}\right) G\left(m_{T}^{(\delta)}\right) f\left(L_{T}^{(\delta)}\right) k\left(\rho_{T}^{(\delta)}\right) 1_{m_{T}^{(\delta)}=S_{T}^{(\delta)}}\right.} \\
& =\int_{\mathbb{R}_{+}^{2}} \frac{f(u) u^{1 / \mu} e^{-u \operatorname{coth} z} G(z) F(z) d u d z}{\Gamma\left(\frac{1}{\mu}\right)(\operatorname{sh} z)^{1+1 / \mu}} \int_{0}^{z} k(x) \frac{\operatorname{sh}(z-x)}{\operatorname{sh} z} d x .
\end{aligned}
$$

Now, Proposition 5.10 appears as an immediate consequence of Proposition 5.11 and of formula (5.5). See Section A.1 for the proof of Proposition 5.11.

5.5. We now present some explicit computations which we deduce from our preceding results.

Proposition 5.12. The law of the 4-tuple $\left(S_{T}^{(\delta)}, m_{T}^{(\delta)}, L_{T}^{(\delta)}, \rho_{T}^{(\delta)}\right)$ is given by:

the variable $L_{T}^{(\delta)} \operatorname{coth}\left(m_{T}^{(\delta)}\right)$ is independent from the triplet $\left(m_{T}^{(\delta)}, S_{T}^{(\delta)}, \rho_{T}^{(\delta)}\right)$, and

$$
\begin{aligned}
& L_{T}^{(\delta)} \operatorname{coth}\left(m_{T}^{(\delta)}\right) \stackrel{\text { law }}{=} G_{\delta}, \\
& \mathbb{P}\left[m_{T}^{(\delta)}<S_{T}^{(\delta)} ; S_{T}^{(\delta)} \in d t, m_{T}^{(\delta)} \in d s, \rho_{T}^{(\delta)} \in d x\right]=\frac{(\delta-1)^{2}(\operatorname{sh} s)^{\delta-2}}{(\operatorname{ch} s)^{\delta}(\operatorname{sh} t)^{\delta}} \operatorname{sh} x 1_{0<x<t} 1_{0<s<t} d s d t d x
\end{aligned}
$$

and,

$$
\mathbb{P}\left[m_{T}^{(\delta)}=S_{T}^{(\delta)} ; m_{T}^{(\delta)} \in d s, \rho_{T}^{(\delta)} \in d x\right]=\frac{(\delta-1) \operatorname{sh}(s-x)}{\operatorname{sh} s(\operatorname{ch} s)^{\delta}} 1_{0<x<s} d x d s .
$$

Identities (5.18) and (5.20) are immediate consequences of formulae (5.16) and (5.17). For the proof of (5.19), see Appendix (A.2). 
In fact this last result may be (partially) obtained using Lemma 4.5 of [7], and following the method we develop in this paper.

Remark 5.13. We may write, thanks to (5.14):

$$
\mathbb{E}\left[F\left(S_{T}^{(\delta)}\right) f\left(L_{T}^{(\delta)}\right) k\left(\rho_{T}^{(\delta)}\right) 1_{m_{T}^{(\delta)}<S_{T}^{(\delta)}}\right]=\mathbb{E}\left[F\left(S_{T}^{(\delta)}\right) f\left(G_{\delta-1} \operatorname{th}\left(S_{T}^{(\delta)}\right)\right) k\left(\rho_{T}^{(\delta)}\right) \frac{\operatorname{th}\left(\rho_{T}^{(\delta)}\right)}{\operatorname{th}\left(S_{T}^{(\delta)}\right)}\right] .
$$

Then, we can compute the right-hand side thanks to the form of the density (1.6) given in Theorem 1.3, and the left-hand side thanks to formula (5.16), jointly with the scaling property of perturbed Brownian motion. So, we may obtain interesting formulae which relate $\left|B_{1}\right|, X_{1}^{\mu}$ and $S_{1}^{\mu}$ (see also remark (A.5)).

\section{Acknowledgement}

We would like to thank an anonymous referee for many helpful suggestions which allowed us to improve this paper.

\section{Appendix A}

\section{A.1. Proof of Proposition 5.11}

From now on, we fix positive measurable functions $F, G, f$ and $k$.

Lemma A.1. Define $A_{t}^{F, k}=\int_{0}^{t} F\left[\mu l_{s}^{0}(B)\right] k\left[X_{s}^{\mu /-}\right] e^{-s / 2} 1_{X_{s}^{\mu}<0} d s$, and $\theta_{t}=\left(A_{t}^{F, k}, t,\left|B_{t}\right|, l_{t}^{0}(B)\right)$. Let $\tau_{u}^{\mu}=$ $\inf \left\{s ; l_{s}^{\mu}>u\right\}$ be the right continuous inverse of $l^{\mu}=l^{0}\left(X^{\mu}\right)$, the local time at 0 of the perturbed Brownian motion $X^{\mu}$. Then $\left(\theta_{t} ; t \geqslant 0\right)$ and $\left(\theta_{\tau_{u}} ; u \geqslant 0\right)$ are Markov processes.

Lemma A.2. For any $y_{i}=\left(a_{i}, s_{i}, u_{i}, v_{i}\right), i \in\{1,2\}$, define $H_{y_{1}}\left(y_{2}\right)=H\left(y_{1}, y_{2}\right)=\left(a_{2}-a_{1}\right) e^{\frac{1}{2} s_{1}}$. Then:

$$
\left[F\left(S_{T}^{(\delta)}\right) G\left(m_{T}^{(\delta)}\right) f\left(L_{T}^{(\delta)}\right) k\left(\rho_{T}^{(\delta)}\right)\right]=\mathbb{E}\left[\int_{0}^{\infty} \psi\left(u,\left|B_{\tau_{u}^{\mu}}\right|, A_{\tau_{u}^{\mu}}^{-, \mu}\right) \mathcal{L} H_{\theta_{\tau_{u}^{\mu}}^{\mu}}\left(\theta_{\tau_{u}^{\mu}}\right) d u\right],
$$

where $\mathcal{L}$ is the generator of the Markov process $\left(\theta_{\tau_{u}^{\mu}} ; u \geqslant 0\right)$ and $\psi(u, b, a)=\frac{1}{2} f\left(\frac{u}{2}\right) G(b) e^{-a / 2}$.

Proof. First, we recall $l^{\mu}=l^{0}\left(X^{\mu}\right)$ and $l=l^{0}(B)$ are the local times respectively of $X^{\mu}$ and $B$ at level 0.

$$
\begin{aligned}
& \mathbb{E}\left[F\left(S_{T}^{(\delta)}\right) G\left(m_{T}^{(\delta)}\right) f\left(L_{T}^{(\delta)}\right) k\left(\rho_{T}^{(\delta)}\right)\right] \\
& =\frac{1}{2} \mathbb{E}\left[\int_{0}^{\infty} e^{-t / 2} F\left(\mu l_{\alpha_{t}^{-, \mu}}\right) G\left(\mu l_{g_{\alpha_{t}}^{\mu}, \mu}\right) f\left(\frac{1}{2} l_{\alpha_{t}^{-, \mu}}^{\mu}\right) k\left(X_{\alpha_{t}^{-, \mu}}^{\mu /-}\right) d t\right] \\
& =\frac{1}{2} \mathbb{E}\left[\int_{0}^{\infty} \exp \left(-\frac{1}{2} A_{s}^{-, \mu}\right) F\left(\mu l_{s}\right) G\left(\mu l_{g_{s}^{\mu}}\right) f\left(\frac{1}{2} l_{s}^{\mu}\right) k\left(X_{s}^{\mu /-}\right) 1_{X_{s}^{\mu}<0} d s\right]
\end{aligned}
$$

from the change of variables $t=A_{s}^{-, \mu}$ 


$$
\begin{aligned}
= & \frac{1}{2} \mathbb{E}\left[\sum_{u>0} \int_{\tau_{u-}^{\mu}}^{\tau_{u}^{\mu}} \exp \left(-\frac{1}{2} A_{s}^{-, \mu}\right) F\left(\mu l_{s}\right) G\left(\mu l_{g_{s}^{\mu}}^{\mu}\right) f\left(\frac{1}{2} l_{s}^{\mu}\right) k\left(X_{s}^{\mu /-}\right) 1_{X_{s}^{\mu}<0} d s\right] \\
& =\mathbb{E}\left[\sum_{u>0} \frac{1}{2} f\left(\frac{u}{2}\right) G\left(\mu l_{\tau_{u-}^{\mu}}\right)\left(A_{\tau_{u}^{\mu}}^{F, k}-A_{\tau_{u-}^{\mu}}^{F, k}\right)\right] \\
& =\mathbb{E}\left[\sum_{u>0} \psi\left(u, \mu l_{\tau_{u-}^{\mu}}, A_{\tau_{u-}^{\mu}}^{-, \mu}\right) H\left(\theta_{\tau_{u-}^{\mu}}^{\mu}, \theta_{\tau_{u}^{\mu}}^{\mu}\right) 1_{\theta_{\tau_{u-}^{\mu}}^{\mu} \neq \theta_{\tau_{u}^{\mu}}}\right] .
\end{aligned}
$$

The result follows, since $\mu l_{\tau_{u-}^{\mu}}=\left|B_{\tau_{u-}^{\mu}}\right|,\left(\psi\left(u, \mu l_{\tau_{u-}^{\mu}}, A_{\tau_{u-}^{\mu}}^{-, \mu}\right)\right)_{u>0}$ is $\left(\mathcal{F}_{\tau_{u}^{\mu}}\right)_{u>0}$-predictable, and $\left(\theta_{\tau_{u}^{\mu}} ; u \geqslant 0\right)$ is a Markov process, to which we apply the general compensation formula (see [33]).

Since the components $(a, s, u, v)$ of $\theta_{\tau_{t}^{\mu}}$ are linked by $u=\mu v$, we only need, in order to deduce formulae (5.16) and (5.17) from (A.1), to obtain the following (partial) explicit expression for the generator $\mathcal{L}$.

Lemma A.3. For $y=\left(a, s, u, \frac{u}{\mu}\right)$, we have

$$
\mathcal{L} H_{y}(y)=h(u),
$$

where

$$
\begin{aligned}
h(u)=F(u) & \left\{\int_{0}^{\infty} e^{-y} k(y) d y-\frac{u^{2}}{2 \operatorname{sh} u} \mathbb{E}\left[\int_{0}^{T_{1}(B)} k\left[u\left(1-B_{x}\right)\right] e^{-u^{2} x / 2} d x\right]\right\} \\
& +\frac{u^{2}}{2 \operatorname{sh} u} \mathbb{E}\left[\int_{0}^{T_{1}\left(X^{\mu}\right)} F\left[u\left(1+\mu l_{x}^{0}(B)\right)\right] k\left[u\left(1-X_{x}^{\mu}\right)\right] e^{-u^{2} x / 2} d x\right] .
\end{aligned}
$$

We assume Lemma A.3 for the moment. We recall the following consequence of the Ray-Knight Theorem 3.2 of [7] about the perturbed Brownian motion:

$$
\left(\left|B_{\tau_{1}^{\mu}}\right|, A_{\tau_{1}^{\mu}}^{-, \mu}\right) \stackrel{\text { law }}{=}\left(T_{0}, \int_{0}^{T_{0}} V_{x} d x ; \text { under } \mathbb{Q}_{1}^{2-2 / \mu}\right),
$$

where $\left(V_{x} ; x \leqslant T_{0}\right)$ is the square of a $(2-2 / \mu)$-dimensional Bessel process, starting at 1 , and considered until $T_{0}$, the first time it reaches 0 . Thus, by scaling:

$$
\left(\left|B_{\tau_{t}^{\mu}}\right|, A_{\tau_{t}^{-, \mu}}^{-, \mu} \stackrel{\text { law }}{=}\left(t T_{0}, t^{2} \int_{0}^{T_{0}} V_{x} d x, \text { under } \mathbb{Q}_{1}^{2-2 / \mu}\right) .\right.
$$

Classical computations on squares of Bessel processes (see [42]) imply:

$$
\mathbb{E}\left[f\left(L_{T}^{(\delta)} \operatorname{coth}\left(S_{T}^{(\delta)}\right)\right) F\left(S_{T}^{(\delta)}\right) k\left(\rho_{T}^{(\delta)}\right) 1_{S_{T}^{(\delta)}=m_{T}^{(\delta)}}\right]=\mathbb{E}\left[f\left(G_{1+1 / \mu}\right)\right] \frac{1}{\mu} \int_{0}^{\infty} \frac{F(z) d z}{(\operatorname{ch} z)^{1+1 / \mu}}\{\ldots\}
$$

where

$$
\{\ldots\}=\int_{0}^{\infty} e^{-y} k(y) d y-\frac{z^{2}}{2 \operatorname{sh} z}\left[\int_{0}^{T_{1}(B)} k\left(z\left(1-B_{x}\right)\right) e^{-z^{2} x / 2} d x\right] .
$$


Comparing this formula, in the case $\delta=2$ to the known results in Corollary 5.8 and Lemma 5.7, we deduce:

$$
\{\ldots\}=\int_{0}^{z} k(x) \frac{\operatorname{sh}(z-x)}{\operatorname{sh} z} d x
$$

Then, reinjecting identity (A.3) in expression (A.2) jointly with the law of the pair $\left(S_{T}^{(\delta)}, \rho_{T}^{(\delta)}\right)$ given in Theorem 5.4, we obtain the identity (5.14). Identity (5.15) then follows from (5.14) and Theorem 5.4.

Proof of Lemma A.3. Let us notice first that, if $\left(P_{t}\right)_{t \geqslant 0}$ is the semi-group of the Markov process $\left(\theta_{\tau_{t}^{\mu}} ; t \geqslant 0\right)$, we have, for any $t>0$ :

$$
\Delta_{t}(y) \stackrel{\text { def }}{=} \frac{P_{t} H_{y}(y)-H_{y}(y)}{t}=\frac{1}{t} \mathbb{E}_{u}\left[\int_{0}^{\tau_{t}^{\mu, u}} F\left(u+\mu l_{w}\right) k\left(u-X_{w}^{\mu}\right) 1_{X_{w}^{\mu}<u} e^{-w / 2} d w\right],
$$

where $\tau_{t}^{\mu, u}$ is the inverse of $l^{\mu, u}$, the local time of the perturbed Brownian motion $X^{\mu}$ at level $u$, and where $\mathbb{E}_{u}$ means that the Brownian motion which appears in the definition of $X^{\mu}$ starts at $u$.

We decompose the latter expectation in two terms corresponding with $\left\{T_{0}(B)>\tau_{t}^{\mu, u}\right\}$ or $\left\{T_{0}(B) \leqslant \tau_{t}^{\mu, u}\right\}$; on the latter set, we have: $\tau_{t}^{\mu, u}=T_{0}(B)+\hat{\tau}_{t-l_{T_{0}(B)}^{\mu, u}}^{u}$, where $\hat{\tau}^{\mu, u}$ is the inverse of the local time at level $u$ of a $\mu$-process $\hat{X}^{\mu}=\hat{B}-\mu \hat{l}$ built from a Brownian motion $\hat{B}$ independent from $\mathcal{F}_{T_{0}}$. Then, noticing that $l_{T_{0}(B)}^{\mu, u}=l_{T_{0}(B)}^{u}$ :

$$
\begin{aligned}
\Delta_{t}(y)= & \frac{1}{t} \mathbb{E}_{u}\left[\int_{0}^{T_{0}(B) \wedge \tau_{t}^{\mu, u}} F(u) k\left(u-B_{w}\right) 1_{B_{w}<u} e^{-w / 2} d w\right] \\
& +\frac{1}{t} \mathbb{E}_{u}\left[1_{l_{T_{0}(B)}^{u} \leqslant t} e^{-\frac{T_{0}(B)}{2}} \int_{0}^{\hat{\tau}_{t-l}^{\mu, u} l_{T_{0}(B)}^{u}} F\left(u+\mu \hat{l}_{w}\right) k\left(u-\hat{X}_{w}^{\mu}\right) 1_{\hat{X}_{w}^{\mu}<u} e^{-w / 2} d w\right] .
\end{aligned}
$$

The second term of the right hand side of Eq. (A.5) is equal to:

$$
\begin{aligned}
& \frac{1}{t} \int_{0}^{t} \mathbb{P}_{u}\left[e^{-\frac{T_{0}(B)}{2}} l_{T_{0}(B)}^{u} \in d s\right] \mathbb{E}_{0}\left[\int_{0}^{\hat{t}_{t-s}^{\mu, u}} F\left(u+\mu \hat{l}_{w}\right) k\left(u-\hat{X}_{w}^{\mu}\right) 1_{\hat{X}_{w}^{\mu}<u} e^{-w / 2} d w\right] \\
& \quad=\frac{1}{t} \int_{0}^{t} \frac{d s}{2 \operatorname{sh} u} \exp \left(-\frac{s}{2}(1+\operatorname{coth} u)\right) \mathbb{E}_{0}\left[\int_{0}^{\hat{\tau}_{t-s}^{\mu, u}} F\left(u+\mu \hat{l}_{w}\right) k\left(u-\hat{X}_{w}^{\mu}\right) 1_{\hat{X}_{w}^{\mu}<u} e^{-w / 2} d w\right] .
\end{aligned}
$$

Since $\lim _{\varepsilon \rightarrow 0} \hat{\tau}_{\varepsilon}^{\mu, u}=T_{u}\left(\hat{X}^{\mu}\right)$, the first time $\hat{X}^{\mu}$ reaches $u$, the above quantity converges, as $t$ goes to 0 , towards

$$
\begin{aligned}
& \frac{1}{2 \operatorname{sh} u} \mathbb{E}_{0}\left[\int_{0}^{T_{u}\left(\hat{X}^{\mu}\right)} F\left(u+\mu \hat{l}_{w}\right) k\left(u-\hat{X}_{w}^{\mu}\right) 1_{\hat{X}_{w}^{\mu}<u} e^{-w / 2} d w\right] \\
& =\frac{u^{2}}{2 \operatorname{sh} u} \mathbb{E}_{0}\left[\int_{0}^{T_{1}\left(X^{\mu}\right)} F\left[u\left(1+\mu l_{w}\right)\right] k\left[u\left(1-X_{w}^{\mu}\right)\right] 1_{X_{w}^{\mu}<1} e^{-w u^{2} / 2} d w\right]
\end{aligned}
$$

by scaling. 
By scaling again, the first term of the right member of (A.5) has the same limit as the following one, when $t$ tends to 0 :

$$
u F(u) \frac{1}{t} \mathbb{E}_{1}\left[\int_{0}^{T_{0}(B) \wedge \tau_{t}^{\mu, 1}} k\left[u\left(1-B_{w}\right)\right] 1_{B_{w}<1} e^{-w u^{2} / 2} d w\right] \stackrel{\text { def }}{=} u F(u) I_{t}(y) .
$$

As $T_{0}(B) \wedge \tau_{t}^{\mu, 1}=T_{0}(B) \wedge \tau_{t}^{(1)}$, where $\tau^{(1)}$ is the inverse of the local time, at level 1, of the Brownian motion $B$ starting at 1 , we have:

$$
\begin{aligned}
I_{t}(y) & =\frac{1}{t} \mathbb{E}_{1}\left[\int_{0}^{T_{0}(B) \wedge \tau_{t}^{(1)}} k\left[u\left(1-B_{w}\right)\right] 1_{B_{w}<1} e^{-w u^{2} / 2} d w\right] \\
& =\frac{1}{t} \mathbb{E}_{0}\left[\int_{0}^{T_{1}(B) \wedge \tau_{t}} k\left[u B_{w}\right] 1_{B_{w}>0} e^{-w u^{2} / 2} d w\right] \\
& =\frac{1}{t} \mathbb{E}_{0}\left[\int_{0}^{\tau_{t}} k\left[u B_{w}\right] 1_{B_{w}>0} e^{-w u^{2} / 2} d w\right]-\frac{1}{t} \mathbb{E}_{0}\left[1_{\tau_{t}>T_{1}(B)} \int_{T_{1}(B)}^{\tau_{t}} k\left[u B_{w}\right] 1_{B_{w}>0} e^{-w u^{2} / 2} d w\right] .
\end{aligned}
$$

Thanks to excursion theory, the first term in (A.7) converges to $\frac{1}{u} \int_{0}^{\infty} k(w) e^{-w} d w$. For the second one, we write $\tau_{t}=T_{1}(B)+\hat{\tau}_{t-l_{T_{1}(B)}}$ and we proceed as before. This second term converges to

$$
\frac{u}{\operatorname{sh} u} \mathbb{E}_{1}\left[\int_{0}^{T_{0}(B)} k\left(u B_{w}\right) 1_{B_{w}>0} e^{-u^{2} w / 2} d w\right]=\frac{u}{\operatorname{sh} u} \mathbb{E}_{0}\left[\int_{0}^{T_{1}(B)} k\left[u\left(1-B_{w}\right)\right] e^{-u^{2} w / 2} d w\right] .
$$

To conclude, we have obtained:

$$
\begin{aligned}
\mathcal{L} H_{y}(y)= & \lim _{t \rightarrow 0} \Delta_{t}(y) \\
= & \frac{u^{2}}{2 \operatorname{sh} u} \mathbb{E}_{0}\left[\int_{0}^{T_{1}\left(X^{\mu}\right)} F\left[u\left(1+\mu l_{w}\right)\right] k\left[u\left(1-X_{w}^{\mu}\right)\right] 1_{X_{w}^{\mu}<1} e^{-w u^{2} / 2} d w\right] \\
& +F(u)\left[\int_{0}^{\infty} k(w) e^{-w} d w-\frac{u^{2}}{\operatorname{sh} u} \mathbb{E}_{0}\left[\int_{0}^{T_{1}(B)} k\left[u\left(1-B_{w}\right)\right] e^{-u^{2} w / 2} d w\right]\right] .
\end{aligned}
$$

\section{A.2. Sketch of the proof of identity (5.19)}

We first recall (see [9]) that if, for $\alpha<1$ and $\beta<1$, the $(\alpha, \beta)$-doubly perturbed Brownian motion $Y^{\alpha, \beta}$ is the unique solution of (with obvious notations):

$$
Y_{t}^{\alpha, \beta}=B_{t}+\alpha S_{t}^{\alpha, \beta}-\beta I_{t}^{\alpha, \beta},
$$

we have, denoting by $T_{v}$ an independent exponential time of parameter $\frac{v^{2}}{2}(v>0): \forall a \in \mathbb{R}, \forall b>0, \forall c>0$, 


$$
\begin{aligned}
\mathbb{E}\left[\exp \left(i a Y_{T_{v}}^{\alpha, \beta}-b S_{T_{v}}^{\alpha, \beta}-c I_{T_{v}}^{\alpha, \beta}\right)\right] & =v^{2} \int_{0}^{\infty} s \exp \left(-\frac{v^{2} s^{2}}{2}\right) \mathbb{E}\left[\exp \left(s\left(i a Y_{1}^{\alpha, \beta}-b S_{1}^{\alpha, \beta}-c I_{1}^{\alpha, \beta}\right)\right)\right] d s \\
& =\frac{v^{2}}{v^{2}+a^{2}}\left\{1+F_{v}(\alpha, \beta, a, b, c)+F_{v}(\beta, \alpha,-a, c, b)\right\},
\end{aligned}
$$

with

$$
\begin{aligned}
F_{v}(\alpha, \beta, a, b, c)= & \frac{(i a \alpha-b) B\left(\frac{\beta}{2}+\frac{c}{2 v} ; 2-\alpha-\beta\right)}{(b-i a+v(1-\alpha)) B\left(1-\beta ; \frac{\beta}{2}+\frac{c}{2 v}\right)} \\
& .3 F_{2}\left(1-\alpha, \frac{b-i a}{2 v}+\frac{1-\alpha}{2}, \frac{\beta}{2}+\frac{c}{2 v} ; \frac{b-i a}{2 v}+\frac{3-\alpha}{2}, 2-\alpha-\frac{\beta}{2}+\frac{c}{2 v} ; 1\right),
\end{aligned}
$$

where ${ }_{3} F_{2}\left(a_{1}, a_{2}, a_{3} ; b_{1}, b_{2} ; z\right)$ are hypergeometric functions (see [32]). Let $\alpha>0, \beta>0, \lambda \in \mathbb{R}$. Thanks to (5.16), we have, always with the same scaling arguments $\left(\delta=1+\frac{1}{\mu}\right)$ :

$$
\begin{aligned}
\mathbb{E} & {\left[1_{m_{T}^{(\delta)}<S_{T}^{(\delta)}} \operatorname{sh}\left(m_{T}^{(\delta)}\right)\left(\operatorname{ch}\left(m_{T}^{(\delta)}\right)\right)^{\delta} e^{-\beta\left(S_{T}^{(\delta)}-m_{T}^{(\delta)}\right)} e^{-\alpha m_{T}^{(\delta)}} e^{i \lambda \rho_{T}^{(\delta)}}\right] } \\
& =\mathbb{E}\left[\int_{\mathbb{R}_{+}^{2}} \frac{d z d x}{2 \mu} z^{2} e^{-\alpha z} 1_{\sqrt{x} S_{1}^{\mu}<1} e^{i \lambda z\left(1-\sqrt{x} X_{1}^{\mu}\right)} e^{-\beta z \sqrt{x} \mu l_{1}} e^{-x z^{2} / 2}\right] \\
& =\int_{0}^{\infty} \frac{s d s}{(\alpha-i \lambda) \mu} e^{-s^{2} / 2} \mathbb{E}\left[e^{-i \lambda s X_{1}^{\mu}} e^{-(\alpha-i \lambda) s S_{1}^{\mu}} e^{-\beta s \mu l_{1}}\right] \\
& =\int_{0}^{\infty} \frac{s d s}{(\alpha-i \lambda) \mu} e^{-s^{2} / 2} \mathbb{E}\left[e^{-i \lambda s X_{1}^{\mu}} e^{-(\alpha-i \lambda) s S_{1}^{\mu}} e^{-\beta s I_{1}^{\mu}}\right],
\end{aligned}
$$

where $I_{t}^{\mu}=\sup _{s \leqslant t}\left(-X_{s}^{\mu}\right)$. As $X^{\mu}=Y^{0,1-1 / \mu}$ (see (1.2) or [9]), we can use (A.10). The rest of the proof is just technical computations on special functionals made with the help of Lebedev [32], more precisely, formula (9.3.4), p. 244, formulae 5.11(1) and 5.11(5) p. 261, and exercise 4, p. 277.

We then obtain:

$$
\begin{aligned}
& \mathbb{E}\left[1_{m_{T}^{(\delta)}<S_{T}^{(\delta)}} \operatorname{sh}\left(m_{T}^{(\delta)}\right)\left(\operatorname{ch}\left(m_{T}^{(\delta)}\right)\right)^{\delta} e^{-\beta\left(S_{T}^{(\delta)}-m_{T}^{(\delta)}\right)} e^{-\alpha m_{T}^{(\delta)}} e^{i \lambda \rho_{T}^{(\delta)}}\right] \\
& =\int_{\mathbb{R}_{+}^{3}} \frac{2 e^{-\beta t} e^{-\alpha s} e^{i \lambda x}}{\mu^{2}\left(1-e^{-2(s+t)}\right)^{1+\frac{1}{\mu}}} \operatorname{sh} x e^{-(s+t)} e^{-\frac{t}{\mu}}\left(1-e^{-2 s}\right)^{\frac{1}{\mu}} 1_{x<s+t} d s d t d x .
\end{aligned}
$$

Remark A.4. In particular, combining (A.11) (with $v=1$ ) and (A.12), thanks to some integrations by parts, we prove Proposition 1.5.

Remark A.5. In the particular case $\mu=1$, we recover from Proposition 1.5 the following results (see $[12,47]$ and [53]):

(i)

$$
\begin{aligned}
\mathbb{P} & \left.S_{T} \leqslant x, I_{T} \leqslant y, B_{T} \in d a\right] \\
& =\frac{1}{2}\left(e^{-|a|}-\frac{\operatorname{sh} y}{\operatorname{sh}(x+y)} e^{-|a-x|}-\frac{\operatorname{sh} x}{\operatorname{sh}(x+y)} e^{-|a+y|}\right) d a, \quad-y \leqslant a \leqslant x,
\end{aligned}
$$


(ii)

$$
\mathbb{P}\left[S_{T}-B_{T} \leqslant u, I_{T}+B_{T} \leqslant v\right]=\mathbb{P}\left[S_{T} \leqslant u, I_{T} \leqslant v\right]=1-\frac{\operatorname{sh} u+\operatorname{sh} v}{\operatorname{sh}(u+v)}
$$

As an application, one can prove that the distribution function of the ratio $\frac{S_{1}}{S_{1}+I_{1}} \stackrel{\text { law }}{=} \frac{S_{1}-B_{1}}{S_{1}+I_{1}}$ admits both the continuous integral and discrete representations (see [14] and [44]):

$$
\begin{aligned}
\mathbb{P}\left[\frac{S_{1}}{S_{1}+I_{1}} \leqslant a\right] & =\frac{1-a}{2} \int_{0}^{+\infty} \frac{\operatorname{sh}(a x)}{\left(\operatorname{ch} \frac{x}{2}\right)^{2}} d x \quad(0<a<1) \\
& =(1-a)\left\{2 a \sum_{n \geqslant 1} \frac{(-1)^{n-1}}{n+a}+\frac{\pi a}{\sin (\pi a)}-1\right\} \\
& =2 a(1-a) \sum_{n \geqslant 1} \frac{(-1)^{n-1} n}{n^{2}-a^{2}} .
\end{aligned}
$$

In the general case, one can prove from (1.8) that:

\begin{tabular}{|c|c|}
\hline$X^{\mu}$ & $\begin{array}{l}\mu \text {-perturbed Brownian motion or } \mu \text {-process } \\
\text { (see Section 1.1) }\end{array}$ \\
\hline$\rho_{t}^{(\delta)}$ & $\begin{array}{l}(2-\delta) \text {-perturbed reflected Brownian motion } \\
\text { (see Eq. (1.3)) }\end{array}$ \\
\hline$\rho_{t}=\rho_{t}^{(2)}$ & $\begin{array}{l}\text { reflected Brownian motion } \\
\text { (see Theorem } 1.1 \text { and Section 5.3) }\end{array}$ \\
\hline$Y^{(c)}$ & $\begin{array}{l}\text { bang-bang diffusion with parameter } c \\
\text { (see Section 2.3, Example 3) }\end{array}$ \\
\hline$\tilde{\rho}^{(c)}$ & $\begin{array}{l}\text { absolute value of the bang-bang diffusion } \\
\text { (see Section 2.3, Example 3) }\end{array}$ \\
\hline$\hat{B}^{(c)}$ & $\begin{array}{l}\text { Brownian motion with drift } c \\
\text { (see Section 2.3, Example 3) }\end{array}$ \\
\hline$R^{(2, c)}$ & $\begin{array}{l}\text { 2-dimensional Bessel process with "naïve" drift } c \\
\text { (see Section 2.3, Example 3) }\end{array}$ \\
\hline$Y^{\alpha, \beta}$ & $\begin{array}{l}(\alpha, \beta) \text {-doubly perturbed Brownian motion } \\
\text { (see Section A.2) }\end{array}$ \\
\hline
\end{tabular}

$$
\begin{aligned}
\mathbb{P}\left[\frac{1}{\left(S_{1}^{\mu}+I_{1}^{\mu}\right)^{2}}, \frac{S_{1}^{\mu}}{S_{1}^{\mu}+I_{1}^{\mu}} \in d s\right] & =\frac{s^{\frac{1}{\mu}-1}}{2 \mu}\left(s+\frac{1-s}{\mu}\right) 1_{0<s<1} d s \\
& =\frac{1}{2} 1_{0<s<1} d s \quad \text { when } \mu=1 .
\end{aligned}
$$

Table A.1

Notations for some processes considered in this paper

\section{References}

[1] V.E. Benes, L.A. Shepp, H.S. Witsenhausen, Some solvable stochastic control problems, Stochastics 4 (1) (1980) 39-83.

[2] J. Bertoin, W. Werner, Comportement asymptotique du nombre de tours effectués par la trajectoire brownienne plane, in: Sém. Probab. XXVIII, in: Lect. Notes Math., vol. 1583, Springer, Berlin, 1994, pp. 164-171. 
[3] J. Bertoin, W. Werner, Asymptotic windings of planar Brownian motion revisited via the Ornstein Uhlenbeck process, in: Sém. Probab. XXVIII, in: Lect. Notes Math., vol. 1583, Springer, Berlin, 1994, pp. 138-152.

[4] Ph. Biane, M. Yor, Sur la loi des temps locaux browniens pris en un temps exponentiel, in: Sém. Probab. XXIII, in: Lect. Notes Math., vol. 1321, Springer, Berlin, 1988, pp. 454-466.

[5] Ph. Biane, M. Yor, Valeurs principales associées aux temps locaux browniens, Bull. Sci. Maths. 2ème Sér. 111 (1987) $23-101$.

[6] A. Borodin, P. Salminen, Handbook of Brownian Motion: Facts and Formulae, second ed., Birkhaüser, 2002.

[7] Ph. Carmona, F. Petit, M. Yor, Some extensions of the arcsine law as (partial) consequences of the scaling property of Brownian motion, Probab. Theory Related Fields 100 (1994) 1-29.

[8] Ph. Carmona, F. Petit, M. Yor, An identity in law involving reflecting Brownian motion, derived from generalized arc-sine laws for perturbed Brownian motions, Stochastic Process. Appl. 7 (1999) 323-333.

[9] Ph. Carmona, F. Petit, M. Yor, Beta variables as time spent in [0, $\infty$ [ by certain perturbed Brownian motions, J. London Math. Soc. (2) 58 (1999) 239-256.

[10] Ph. Carmona, F. Petit, J. Pitman, M. Yor, On the laws of homogeneous functionals of the Brownian bridge, in: Studia Scientiarum Mathematicarum Hungarica, vol. 35, 1999, pp. 445-455.

[11] L. Chaumont, R.A. Doney, Pathwise uniqueness for perturbed versions of Brownian motion and reflected Brownian motion, Probab. Theory Related Fields 113 (nf 4) (1999) 519-534.

[12] L. Chaumont, M. Yor, Some Exercices in Probability, Cambridge University Press, 2003.

[13] A.S. Cherny, A.N. Shiryaev, Some properties of Brownian motion with a drift, and a generalization of a theorem of P. Lévy, Teor. Veroyatnost. i Primenen 44 (2) (1999) 466-472. English translation in Theory Probab. Appl. 44 (2) (2000) 412-418.

[14] E. Csaki, On some distributions concerning the maximum and minimum of a Wiener process, in: Proc. Colloq. Methods of Complex Anal. in the Theory of Probab. and Statist., Kossuth L. Univ. Debrecen, Debrecen, 1977, Colloq. Math. Soc. János Bolyai 21 (1979) 43-52.

[15] E. Csaki, A. Földes, On two ergodic theorems for self-similar processes, in: B. Szyszkowicz (Ed.), Asymptotic Methods in Probability and Statistics, a volume in honour of Miklós Csörgő, 1998, pp. 97-111.

[16] M. Csörgő, Z. Shi, M. Yor, Some asymptotic properties of the local time of the uniform empirical process, Bernoulli 5 (6) (1999) 10351058.

[17] J.J. Daudin, M.P. Etienne, P. Vallois, Asymptotic behaviour of the local score of independent and identically distributed random sequences, Stochastic Process. Appl. 107 (nf 1) (2003) 1-28.

[18] B. Davis, Perturbed random walks and Brownian motions, and local times, Proceedings of the New York Journal of Mathematics Conference, New York J. Math. 3A (June 9-13, 1997) 81-87, (electronic).

[19] R.A. Doney, Y.B. Nakhi, Perturbed and non-perturbed Brownian taboo processes, Ann. Inst. H. Poincaré 37 (2001) 725-736.

[20] R.A. Doney, J. Warren, M. Yor, Perturbed Bessel processes, in: Séminaire de Probabilités XXXII, in: Lecture Notes in Math., vol. 1686, Springer, Berlin, 1998, pp. 237-249.

[21] N. Eisenbaum, Un théorème de Ray-Knight lié au supremum des temps locaux, Probab. Theory Related Fields 87 (1990) $79-95$.

[22] P. Fitzsimmons, A converse to a theorem of P. Lévy, Ann. Probab. 15 (1987) 1515-1523.

[23] H. Föllmer, C.T. Wu, M. Yor, On weak Brownian motions of arbitrary order, Ann. Institut H. Poincaré 36 (4) (2000) $447-487$.

[24] T. Fujita, F. Petit, M. Yor, Pricing path-dependent options in some Black-Scholes market, from the distribution of homogeneous Brownian functionals, J. Appl. Probab. 41 (1) (2004).

[25] S.E. Graversen, A.N. Shiryaev, An extension of P. Lévy's distributional properties to the case of a Brownian motion with drift, Bernoulli 6 (4) (2000) 615-620.

[26] Y. Hu, Z. Shi, The limits of Sinaï's simple random walk in random environment, Ann. Probab. 26 (4) (2000) 1477-1521.

[27] T. Jeulin, Application du grossissement de filtration à l'étude des temps locaux du mouvement brownien, in: Lect. Notes Math., vol. 1118, Springer, Berlin, 1985.

[28] I. Karatzas, S. Shreve, Brownian Motion and Stochastic Calculus, second ed., Springer, Berlin, 1991.

[29] G.N. Kinkladze, A note on the structure of processes the measure of which is absolutely continuous with respect to the Wiener process modulus, Stochastics 8 (1982) 39-44.

[30] J.F. Le Gall, M. Yor, Excursions browniennes et carrés de processus de Bessel, C. R. Acad. Sci. Sér. I 303 (1986) 73-76.

[31] J.F. Le Gall, M. Yor, Enlacements du mouvement brownien autour des courbes de l'espace, Trans. Amer. Math. Soc. Sér. I 317 (1990) 687-722.

[32] N.N. Lebedev, Special Functions and Their Applications, Dover Publications, New York, 1972, Translated and edited by Richard A. Silverman.

[33] P.A. Meyer, Intégrales stochastiques IV, in: Sém. Probab. I, in: Lect. Notes Math., vol. 39, Springer, Berlin, 1967, pp. $142-162$.

[34] N. O'Connell, M. Yor, Brownian analogues of Burke's theorem, Stochastic Process. Appl. 96 (2) (2001) 285-304.

[35] M. Perman, An excursion approach to Ray-Knight theorems for perturbed Brownian motion, Stochastic Process. Appl. 63 (1) (1996) $67-74$.

[36] M. Perman, W. Werner, Perturbed Brownian motions, Probab. Theory Related Fields 108 (3) (1997) 357-383.

[37] F. Petit, Sur le temps passé par le mouvement brownien au-dessus d'un multiple de son supremum, et quelques extensions de la loi de l'arcsinus, PhD Thesis, Université Paris VII, 1992. 
[38] F. Petit, Quelques extensions de la loi de l'arcsinus, C. R. Acad. Sci. Sér. I 315 (1992) 855-858.

[39] F. Petit, Document de synthèse pour l'habilitation à diriger des recherches, Laboratoire de Probabilités et Modèles Aléatoires, Université Paris VI, décembre 2003.

[40] F. Petit, M. Yor, Itô's formula and the marginals of certain submartingales, in: A. Shiryaev, A. Sulem (Eds.), Proceedings INRIA, Journées sur les Mathématiques financières (1998) 164-167.

[41] J.W. Pitman, The stochastic differential equations solved by local times of a Brownian excursion or bridge derived from the height profile of a random tree or forest, Ann. Probab. 27 (1) (1999) 261-283.

[42] J.W. Pitman, M. Yor, A decomposition of Bessel Bridges, Z. Wahr. Verw. Geb. 59 (1982) 425-457.

[43] J.W. Pitman, M. Yor, Quelques identités en loi pour les processus de Bessel, in: Astérisque. Hommage à P.A. Meyer et J. Neveu., vol. 236, Société Mathématique de France, 1996, pp. 249-276.

[44] J.W. Pitman, M. Yor, Path decompositions of a Brownian bridge related to the ratio of its maximum and amplitude, Studia Sci. Math. Hungar. 35 (3-4) (1999) 457.

[45] J.W. Pitman, M. Yor, Laplace transforms related to excursions of a one-dimensional diffusion, Bernoulli 5 (2) (1999) $249-255$.

[46] B. Rauscher, Some remarks on Pitman's theorem, in: Sém. Probab. XXXI, in: Lect. Notes Math., vol. 1655, Springer, Berlin, 1997, pp. 266-271.

[47] D. Revuz, M. Yor, Continuous Martingales and Brownian Motion, third ed., Springer, Berlin, 1999.

[48] K. Takaoka, On the martingales obtained by an extension due to Saisho, Tanemura and Yor, of Pitman's theorem, in: Sém. Probab. XXXI, in: Lect. Notes Math., vol. 1655, Springer, 1997, pp. 325-365.

[49] M. Yor, On square-root boundaries for Bessel processes, and pole-seeking Brownian motion, in: A. Truman, D. Williams (Eds.), Stochastic Analysis and Applications, in: Lect. Notes Math., vol. 1095, Springer, Berlin, 1984.

[50] M. Yor, Some Aspects of Brownian Motion. Part I: Some Special Functionals, in: Lect. Math., Birkhaüser, ETH Zürich, 1992.

[51] M. Yor, Random Brownian scaling and some absolute continuity relationships, in: Prog. Probab., vol. 36, Birkhaüser, 1995, pp. $243-252$.

[52] M. Yor, Some Aspects of Brownian Motion. Part II: Some Recent Martingale Problems, in: Lect. Math., Birkhaüser, ETH Zürich, 1997.

[53] M. Yor, Some remarks about the joint law of Brownian motion and its supremum, in: Sém. Probab. XXXI, in: Lect. Notes Math., vol. 1655, Springer, Berlin, 1997, pp. 306-314. 